\title{
LEVI DECOMPOSITIONS OF A LINEAR ALGEBRAIC GROUP
}

\author{
GEORGE J. MCNINCH \\ Dedicated to the memory of Vladimir Morozov and to his contributions to mathematics
}

\begin{abstract}
If $G$ is a connected linear algebraic group over the field $k$, a Levi factor of $G$ is a reductive complement to the unipotent radical of $G$. If $k$ has positive characteristic, $G$ may have no Levi factor, or $G$ may have Levi factors which are not geometrically conjugate. We give in this paper some sufficient conditions for the existence and the conjugacy of Levi factors of $G$.

Let $\mathscr{A}$ be a Henselian discrete valuation ring with fractions $K$ and with perfect residue field $k$ of characteristic $p>0$. Let $G$ be a connected and reductive algebraic group over $K$. Bruhat and Tits have associated to $G$ certain smooth $\mathscr{A}$-group schemes $\mathcal{P}$ whose generic fibers $\mathcal{P} / K$ coincide with $G$; these are known as parahoric group schemes. The special fiber $\mathcal{P}_{/ k}$ of a parahoric group scheme is a linear algebraic group over $k$. If $G$ splits over an unramified extension of $K$, we show that $\mathcal{P}_{/ k}$ has a Levi factor, and that any two Levi factors of $\mathcal{P} / k$ are geometrically conjugate.
\end{abstract}

\section{CONTENTS}

1. Introduction

2. Some preliminaries 3

3. Levi decompositions 6

4. Cohomology and Levi factors 8

5. Some cohomological criteria for existence and conjugacy of Levi factors 11

6. Parahoric group schemes

7. Examples

References

\section{INTRODUCTION}

1.1. Linear groups. Let $G$ be a connected, linear algebraic group over a field $k$, and suppose that the unipotent radical of $G_{/ k_{\text {alg }}}$ is defined over $k$; see $\$ 2.1$. A Levi factor of $G$ is a complement in $G$ to the unipotent radical. If $k$ has characteristic $p>0, G$ may have no Levi factors, or $G$ may non-conjugate Levi factors; see the overview in 83 In this paper, we investigate the existence and conjugacy of Levi factors. For some previous work on these matters, see the paper of J. Humphreys [Hu 67].

Our approach to Levi factors uses results from the cohomology of linear algebraic groups; see 4 For example, suppose that the unipotent radical $R$ of $G$ is a vector group which is $G$-equivariantly isomorphic to a linear representation $V$ of $G$. If $H^{2}(G, V)=0$, then $G$ has a Levi factor, and if $H^{1}(G, V)=0$ then any two Levi factors of $G$ are conjugate by an element of $R(k)=V$. Of course, these results are well-known for "abstract" groups G; see e.g. [Br 94, Ch. IV]. We observe here their validity for a linear algebraic group $G$.

In Theorems 5.1 and 5.2, we give straightforward extensions of these cohomological criteria for the existence and conjugacy of Levi factors when $R$ is no longer a vector group; note that

Date: October 12, 2018.

Research of McNinch supported in part by the US NSA award H98230-08-1-0110. 
our results require $G$ to satisfy condition $(\mathrm{L})$ of $\$ 2.3$, this condition means that $R$ has a filtration for which each consecutive quotient is a vector group with a linear action of $G_{\text {reduc }}$.

For a reductive group $H$ in characteristic $p$, J. C. Jantzen has proved that any $H$-module $V$ having $\operatorname{dim} V \leq p$ is completely reducible; see [Ja 97]. Assume that $\operatorname{dim} R<p$ and that condition (L) holds for $G$. If $G$ has a Levi factor, Jantzen's result implies that any two Levi factors of $G$ are conjugate by an element of $R(k)$. Under a further assumption on the character of the $G_{\text {reduc }}$-module obtained from $R$, Jantzen's result implies the existence of a Levi factor of $G$. For these results, see Theorem 5.3

Finally, if $H$ is reductive and $P$ is a parabolic subgroup, one knows for any linear representation $V$ of $H$ that $H^{i}(H, V) \simeq H^{i}(P, V)$ for $i \geq 0$. Using this fact, we prove for a group $G$ satisfying (L) that the existence of a Levi factor follows from the existence of subgroup of $G$ mapping isomorphically to a Borel subgroup of the reductive quotient $G_{\text {reduc }}$; see the results (5.4.1) and Theorem [5.5] which are crucial to our investigation of Levi factors of the special fibers of parahoric group schemes undertaken in $\$ 6$

1.2. Parahoric group schemes. We wish to apply the conditions just mentioned to the special fiber of a parahoric group scheme; we begin our discussion by briefly describing these group schemes.

Fix a Henselian discrete valuation ring (DVR) $\mathscr{A}$ with fractions $K$ and residues $k$ (recall that if $\mathscr{A}$ is complete, it is Henselian). We suppose the characteristic $p$ of the residue field $k$ to be positive. Moreover, we suppose that $k$ is perfect.

To a connected and reductive group $G$ over $K$, Bruhat-Tits associate a topological space with an action of the group of $K$-rational points $G(K)$; it is known as the affine building $\mathscr{I}$ of $G$. To a facet $\mathcal{F}$ of an apartment of $\mathscr{I}$, there corresponds a smooth affine group scheme $\mathcal{P}_{\mathcal{F}}$ over $\mathscr{A}$ whose generic fiber $\mathcal{P}_{\mathcal{F} / K}$ is equal to $G$; this group scheme has the property that its group of $\mathscr{A}$-points $\mathcal{P}_{\mathcal{F}}(\mathscr{A})$ is the "connected stabilizer" of $\mathcal{F}$ in $G(K)$.

If $G$ is $K$-split and if $\mathcal{F}=\{x\}$ consists in a so-called special point $x$ of $\mathscr{I}$, then the group scheme $\mathcal{P}_{x}$ is split and reductive over $\mathscr{A}$. For general $\mathcal{F}$, the group scheme $\mathcal{P}=\mathcal{P}_{\mathcal{F}}$ is not reductive; in particular, the special fiber $\mathcal{P}_{/ k}$ - the linear algebraic group obtained from $\mathcal{P}$ by base-change to $\operatorname{Spec}(k)$ - need not be reductive over $k$.

In his article in the 1977 Corvallis proceedings [Ti 77, §3.5], J. Tits wrote that the linear group $\mathcal{P}_{/ k}$ possesses a Levi factor. Since the article was intended - as Tits wrote in its opening paragraphs - for utilizers, proofs were mostly omitted. In particular, no proof of this statement was given in loc. cit., and none seems to have appeared elsewhere.

We apply the results of $\$ 5$ to obtain the following results:

Theorem A. Let $\mathcal{P}$ be a parahoric group scheme over $\mathscr{A}$ with generic fiber $G=G_{/ K}$.

(i) If $G$ is split over $K$ and if $S$ is a maximal split torus of $\mathcal{P}_{/ k}$, then $\mathcal{P}_{/ k}$ has a unique Levi factor containing $S$. In particular, any two Levi factors of $\mathcal{P} / k$ are $\mathcal{P}(k)$-conjugate.

(ii) If $G / L$ is split for an unramified extension $K \subset L$, then $\mathcal{P}_{/ k}$ has a Levi factor, and any two Levi factors of $\mathcal{P}_{/ k}$ are geometrically conjugate.

Recall that subgroups (or elements, or ...) are geometrically conjugate under the action of a linear algebraic group $H$ over $k$ provided they are conjugate by an element of $H\left(k_{\mathrm{alg}}\right)$ where $k_{\text {alg }}$ is an algebraic closure of $k$.

We observe that if $G_{/ L}$ is not split for any unramified extension $L \supset K$, the special fiber $\mathcal{P}_{/ k}$ of a parahoric group scheme can possess Levi factors which are not geometrically conjugate; see the example given in $\$ 7.2$

After seeing a preliminary version of this manuscript, Gopal Prasad explained to the author that Rousseau's theorem ([Ro 77] and [Pr 01] ) should allow one to prove the existence of a Levi factor in the special fiber of a parahoric group scheme under the weaker assumption that $G$ splits over a tamely ramified extension of $K$; this result will appear in a subsequent paper. 
1.3. Terminology. By a linear algebraic group $G$ over a field $k$ we mean a smooth, affine group scheme of finite type over $k$. When we speak of a closed subgroup of an algebraic group $G$, we mean a closed subgroup scheme over $k$; thus the subgroup is required to be "defined over $k^{\prime \prime}$ in the language of [Sp 98] or [Bo 91]. Similar remarks apply to homomorphisms between linear algebraic groups. Note that we will occasionally use the terminology " $k$ subgroup" or " $k$-homomorphism" for emphasis. If $\ell \supset k$ is a field extension, we write $G / \ell$ for the linear algebraic group over $\ell$ obtained by extension of scalars. If $g \in G(k)$ is a $k$ rational point of the linear algebraic group $G$, we write $\operatorname{Int}(g)$ for the inner automorphism of $G$ determined by $g$.

Following terminology in [Se 88, §VII.1], we will say that the sequence

$$
1 \rightarrow N \stackrel{i}{\rightarrow} G \stackrel{\pi}{\rightarrow} H \rightarrow 1
$$

of linear algebraic groups is strictly exact if the sequence

$$
1 \rightarrow N\left(k_{\mathrm{alg}}\right) \stackrel{i}{\rightarrow} G\left(k_{\mathrm{alg}}\right) \stackrel{\pi}{\rightarrow} H\left(k_{\mathrm{alg}}\right) \rightarrow 1
$$

is exact for an algebraic closure $k_{\mathrm{alg}}$ of $k$, and if $i$ and $\pi$ are separable so that $\pi$ induces an isomorphism $G / N \rightarrow H$ of algebraic groups.

1.4. Acknowledgments. The author would like to thank B. Conrad, J. Humphreys, J. C. Jantzen, and an anonymous referee for useful remarks on a preliminary version of this manuscript. In particular, I thank the referee for communicating the proof of Proposition 4.2 given here; it is much simpler than the one I originally gave.

\section{SOME PRELIMINARIES}

Throughout this section, $G$ denotes a linear algebraic group over the field $k$; we make further assumptions on $G$ and on $k$ in the final paragraph $\$ 2.4$

2.1. The question of rationality of the unipotent radical over a ground field. The unipotent radical of $G$ is the largest connected, unipotent, normal subgroup $R=R_{u} G_{/ k_{\text {alg }}}$ of $G / k_{\text {alg }}$, where $k_{\text {alg }}$ is an algebraic closure of $k$. As the following example shows, the subgroup $R$ is in general not defined over $k$.

(2.1.1). Let $\ell$ be a purely inseparable extension of $k$ having degree $p$; say $\ell=k(a)$ with $a^{p} \in k$. Consider the k-group $G=R_{\ell / k} \mathbf{G}_{m}$ obtained from the multiplicative group $\mathbf{G}_{m}$ by restriction of scalars. The unipotent radical of the $\ell$-group $G_{/ \ell}$ is defined over $\ell$ and has dimension $p-1$, but any normal, connected, unipotent, smooth subgroup scheme of $G$ defined over $k$ is trivial.

Proof. See [CGP 10, Example 1.1.3], and see e.g. [CGP 10, §A.5] or [Sp 98, 11.4] for the notion of "restriction of scalars".

In what follows, we will often consider the following assumption on $G$.

(R) The unipotent radical $R=R_{u} G$ is defined over $k$.

Note the following:

(2.1.2). If $k$ is perfect, then condition $(\boldsymbol{R})$ holds for the linear group $G$; i.e. $R$ is a $k$-subgroup of $G$.

Proof. This follows by Galois descent; see [Sp 98, 11.2.8, 14.4.5(v)]. 
2.2. Groups with a normal, split unipotent subgroup. Recall [Sp 98, §14] that a connected, unipotent linear group $U$ is said to be $k$-split provided that there is a sequence

$$
1=U_{m} \subset U_{m-1} \subset \cdots \subset U_{1} \subset U_{0}=U
$$

of closed, connected, normal subgroups of $U$ such that each quotient $U_{i} / U_{i+1}$ is isomorphic to $\mathbf{G}_{a / k}$.

(2.2.1) ([Sp 98, 14.3.10]). If $k$ is perfect, every connected unipotent group over $k$ is $k$-split

Definition. A vector group $U$ is a connected, split, abelian, unipotent group having exponent $p$ in case $k$ has characteristic $p>0$.

(2.2.2). The linear algebraic group $U$ is a vector group if and only if it is isomorphic to a direct product of copies of $\mathbf{G}_{a / k}$.

Proof. [Oe 84, V.2.3].

Suppose now that $R \subset G$ is a normal, connected, unipotent subgroup of $G$. By a splitting sequence for $R$ we mean a sequence

$$
1=R_{m} \subset R_{m-1} \subset \cdots \subset R_{1} \subset R_{0}=R
$$

of closed, connected, $k$-subgroups of $R$ which are normal in $G$ with the property that each quotient $R_{i} / R_{i+1}$ is a vector group.

If $R$ possesses a splitting sequence, evidently $R$ is $k$-split. Conversely:

(2.2.3). If $R$ is $k$-split, then $R$ has a splitting sequence.

Proof. Consider first the lower central series where for $i>0, R_{i}=\left(R, R_{i-1}\right)$. Since $R$ is split, it follows from [Sp 98, 14.3.12(3),(2)] that each $R_{i}$ is $k$-split, and that each quotient $R_{i} / R_{i+1}$ is a $k$-split abelian unipotent group. Thus we are reduced to the case where $R$ is abelian $k$-split unipotent. If the characteristic $p$ of $k$ is 0 there is nothing left to do, so suppose $p>0$. If $R$ has exponent $p$, it is a vector group. The result for any commutative $R$ now follows by induction on the exponent by considering the filtration $1 \rightarrow R^{p} \rightarrow R$; note that $R^{p}$ is $k$-split by [Sp 98, 14.3.12(2)].

Proposition. Suppose that the linear algebraic group $G$ has a normal, connected, $k$-split unipotent subgroup $R$. Then there is a morphism of k-varieties $s: G / R \rightarrow G$ which is a section to $\pi$. In particular, $\pi: G(k) \rightarrow(G / R)(k)$ is surjective.

Proof. This is a consequence of results of Rosenlicht [Ro 63]; Lemma 2 of loc. cit. shows that $\pi: G \rightarrow G / R$ is a Zariski-locally trivial principal $R$-bundle, and Theorem 1 of loc. cit. shows, since $G / R$ is affine, that $\pi$ has a regular cross-section defined over $k$.

2.3. Condition (L). Let $U$ be a vector group, and suppose that $G$ acts by group automorphisms on $U$.

Definition. The action of $G$ on $U$ will be said to be linearizable if there is a G-equivariant isomorphism between $U$ and the Lie algebra $\operatorname{Lie}(U)$, where $\operatorname{Lie}(U)$ is viewed as a vector group with its natural action of $G$.

The next result is straightforward:

(2.3.1). The following are equivalent:

(a) The action of $G$ on $U$ is linearizable.

(b) There is a G-equivariant isomorphism $U \simeq V$ for some linear representation $V$ of $G$.

(c) There is an action of the 1 dimensional split torus $\mathbf{G}_{m}$ on $U$ by G-equivariant group automorphisms such that the resulting action on $\operatorname{Lie}(U)$ is scalar multiplication. 
Suppose that condition (R) holds for the linear algebraic group $G$, and that the unipotent radical $R$ of $G$ is $k$-split. For any splitting sequence of $R$ as in (2.2.3), notice that the conjugation action of $R$ on the quotient $R_{i} / R_{i+1}$ is trivial for each $i$; i.e. the reductive quotient $G_{\text {reduc }}=G / R$ acts on each vector group $R_{i} / R_{i+1}$.

We will be interested in the following condition on $G$ :

(L) Condition (R) holds for $G$, the unipotent radical $R$ is $k$-split, and for some splitting sequence of $R$ as in (2.2.3), the $G_{\text {reduc }}$-action on the vector group $R_{i} / R_{i+1}$ is linearizable for each $i$.

A splitting sequence $1=R_{n} \subset R_{n-1} \subset \cdots \subset R_{0}=R$ for which (L) holds will be called a linearizable splitting sequence for $R$.

Remark. (a) If $P$ is a parabolic subgroup of a reductive group $G$, then it is well-known that condition (L) holds for $P$.

(b) Suppose that $G$ is reductive and that $G$ acts by group automorphisms on the vector group $U$. The author is aware neither of a proof that the $G$-action on $U$ is always linearizable, nor of a counterexample to that statement.

(c) The proof of (2.2.3) amounts to a construction of a canonical splitting sequence for which the $R_{i}$ are characteristic in $G$. Nevertheless, we only require the condition in (L) to hold for some splitting sequence; this raises the question: if $k \subset \ell$ is a separable field extension and $(\mathbf{L})$ holds for $G_{/ \ell}$, does (L) hold for $G$ ?

2.4. Recollections: representations of a reductive group. In this section, let $k$ be algebraically closed and let $G$ be a connected and reductive algebraic group over $k$. Fix a maximal torus $T$ of $G$ and a Borel subgroup $B$ containing $T$.

A dominant weight $\tau \in X^{*}(T)$, determines the standard module $H^{0}(\tau)=\operatorname{ind}_{B}^{G}(\tau)$ [Ja 03 , II.2.1] which can be realized as the global sections of a suitable $G$-linearized line bundle on $G / B$. The Weyl module $V(\tau)$ is the contragredient $H^{0}\left(-w_{0} \tau\right)^{\vee}$ [Ja 03, II.2.13] (where $w_{0}$ is the long word in the Weyl group $N_{G}(T) / T$ ).

There is a simple module $L(\tau)$ for which

$$
L(\tau) \simeq \operatorname{soc} H^{0}(\tau) \text { and } L(\tau) \simeq V(\tau) / \operatorname{rad} V(\tau),
$$

where $\operatorname{soc}(-)$ denotes the largest semisimple submodule, and $\operatorname{rad}(-)$ denotes the largest submodule for which the quotient is semisimple; see [Ja 03, II.2.3, II.2.14]. Moreover, if $L$ is a simple $G$-module, then $L$ is isomorphic to $L(\tau)$ for a unique dominant weight $\tau$ [Ja 03. Prop. II.2.4].

We record the following:

(2.4.1) ([Ja 03, Prop. II.2.14]). If $\tau, \gamma \in X^{*}(T)$ are dominant weights for which $\gamma \ngtr \tau$, then

$$
\operatorname{Ext}_{G}^{1}(L(\tau), L(\gamma)) \simeq \operatorname{Hom}_{G}(\operatorname{rad} V(\tau), L(\gamma))
$$

(2.4.2) ([Ja 03, Prop. II.4.16]). If $\tau, \gamma \in X^{*}(T)$ are dominant weights, then

$$
\operatorname{Ext}^{i}\left(V(\tau), H^{0}(\gamma)\right)=0 \text { for all } i>0 \text {. }
$$

In particular, $H^{i}\left(G, H^{0}(\gamma)\right)=0$ for all $i>0$.

For a $G$-module $V$, we may write $V=\bigoplus_{\lambda \in X^{*}(T)} V_{\lambda}$ as a direct sum of its weight spaces; the character of $V$ is then

$$
\operatorname{ch} V=\sum_{\lambda \in X^{*}(T)} \operatorname{dim} V_{\lambda} e^{\lambda}
$$

viewed as an element of the integral group ring $\mathbf{Z}\left[X^{*}(T)\right]$ (which has $\mathbf{Z}$-basis consisting of the $e^{\lambda}$ ). For a dominant weight $\lambda$ we write $\chi(\lambda)=\operatorname{ch} H^{0}(\lambda)=\operatorname{ch} V(\lambda)$; note that $\chi(\lambda)$ is given by Weyl's character formula [Ja 03, II.5.10], so in particular $\operatorname{dim} H^{0}(\lambda)=\operatorname{dim} V(\lambda)$ is given by the Weyl degree formula.

We record: 
(2.4.3) (|Ja 03, II.5.8]). The set $\{\mathrm{ch} L \mid L$ a simple $G$-module $\}$ is a $\mathbf{Z}$-basis for $\mathbf{Z}\left[X^{*}(T)\right]^{W}$.

\section{LEVI DECOMPOSITIONS}

In this section, we discuss the existence and conjugacy of Levi factors in linear algebraic groups over a field $k$. We will write $G$ for a connected linear algebraic group defined over $k$.

3.1. Definitions and first remarks. Assume that (R) holds for $G$.

Definition. A Levi factor of $G$ is a closed $k$-subgroup $M$ of $G$ such that the product mapping

$$
(x, y) \mapsto x y: M \ltimes R \rightarrow G
$$

is a $k$-isomorphism of algebraic groups, where $M \ltimes R$ denotes the semidirect product (for the action of $M$ on $R$ by conjugation).

If there is such a Levi factor $M$, one says that $G$ has a Levi decomposition over $k$. If $M$ is a Levi factor of $G$, then the projection mapping $G \rightarrow G / R$ induces an isomorphism $M \stackrel{\sim}{\rightarrow} G / R$ so that $M$ is connected and reductive.

Suppose for a moment that $k$ has characteristic zero. Apply Levi's theorem [Jac 79, III.9] to the finite dimensional Lie algebra $\mathfrak{g}=\operatorname{Lie}(G)$ to find a semisimple Lie subalgebra $\mathfrak{m} \subset \mathfrak{g}$ such that $\mathfrak{g}=\mathfrak{m} \oplus \mathfrak{r}$ where $\mathfrak{r}$ is the radical of $\mathfrak{g}$. Now, $[\mathfrak{m}, \mathfrak{m}]=\mathfrak{m}$, so that $\mathfrak{m}$ is an algebraic Lie subalgebra by [Bo 91, 7.9]. This condition means that there is a closed connected subgroup $M \subset G$ with $\operatorname{Lie}(M)=\mathfrak{m}$; evidently, $M$ is semisimple. Choosing a maximal torus $T_{0}$ of $M$ and a maximal torus $T$ of $G$ containing $T_{0}$, one finds that M.T is a reductive subgroup of $G$ which is a complement to the unipotent radical $R$. In summary: linear algebraic groups in characteristic zero always have a Levi decomposition. Moreover, it follows from Theorem 5.1 below that any two Levi factors of $G$ are conjugate by an element of the unipotent radical.

On the other hand, if $k$ has positive characteristic, the situation is more complicated.

- $G$ need not have a Levi factor; see 3.2 and 7.1

- Two Levi factors for $G$ need not be geometrically conjugate; see the example given by Borel and Tits in [BoTi 65, 3.15] and see $\$ 7.2$

- Even in the case of an algebraically closed field $k$, in order that a subgroup $M \subset G$ be a Levi factor, it is crucial that multiplication determine an isomorphism $M \ltimes R \rightarrow G$ of algebraic groups and not merely of the "abstract" group of points; see the example in $\$ 3.3$.

3.2. Linear algebraic groups with no Levi decomposition: Witt vector construction. Suppose that $k$ is a perfect field of characteristic $p>0$, let $W(k)$ be the ring of Witt vectors over $k$, and let $W_{2}(k)=W(k) / p^{2} W(k)$ be the ring of length-two Witt vectors [Se 79. II. $\left.\S 6\right]$. One may view $W_{2}(k)$ as a ring-variety over $k$; as a variety, $W_{2}(k)$ is just affine 2-space $\mathbf{A}_{/ k}^{2}$.

Let $G_{/ W_{2}(k)}$ be a split semisimple group scheme over $W_{2}(k)$, and let $G_{/ k}$ be the corresponding semisimple algebraic group obtained by $G_{/ k}=G_{/ W_{2}(k)} \otimes_{W_{2}(k)} k$. Using the point-of-view found in [CGP 10, A.6] - to which we refer for details concerning the following sketch -, we may view the group $G\left(W_{2}(k)\right)$ as a linear algebraic group over $k$ of $\operatorname{dimension} 2 \cdot \operatorname{dim} G / k$. To do this, we note that if $X$ is an affine $W_{2}(k)$-scheme of finite type, the functor on $k$-algebras

$$
\Lambda \mapsto X\left(W_{2}(\Lambda)\right)
$$

is represented by an affine $k$-scheme $X_{2}$ of finite type; the assignment $X \mapsto X_{2}$ is known as the Greenberg functor.

Let $H$ be the value of the Greenberg functor at the smooth affine $W_{2}(k)$-group scheme $G_{/ W_{2}(k)}$. The natural ring homomorphism $W_{2} \rightarrow k$ determines a surjective mapping of algebraic groups $H \rightarrow G_{/ k}$. According to [CGP 10, Lemma A.6.2], the kernel of the mapping $H \rightarrow G_{/ k}$ is a vector group which identifies as a representation for $G_{/ k}$ with the Frobenius twist $\mathfrak{g}^{[1]}$ of the adjoint representation $\mathfrak{g}$. 
(3.2.1). [CGP 10, Prop. A.6.4] The group $H$ has reductive quotient $G_{/ k}$, and $H$ has no Levi decomposition.

Remark. (a) In case $G=\mathrm{SL}_{2}$, one can see also [Mc 03, Remark 5] for a different argument that $\mathrm{SL}_{2}\left(W_{2}\right)$ has no Levi decomposition.

(b) In fact, the example of the 6-dimensional linear group $\mathrm{SL}_{2}\left(W_{2}\right)$ with reductive quotient $\mathrm{SL}_{2 / k}$ and no Levi factor was communicated in 1967 by J. Tits (who learned it from P. Roquette) in a letter to J. Humphreys in connection with Humphreys' paper [Hu 67].

3.3. Complements to the unipotent radical as an abstract group are insufficient. We first recall the following:

(3.3.1) ([Bo 91, 14.19]). Let $P$ be a parabolic subgroup of a reductive group $G$. Then $P$ has a Levi factor defined over $k$, and any two Levi factors of $P$ defined over $k$ are conjugate by a unique element of $R_{u}(P)(k)$.

In defining a Levi factor, the requirement that the product mapping $M \ltimes R \rightarrow G$ be an isomorphism of algebraic groups is essential. Write $\pi: G \rightarrow G / R=G_{\text {reduc }}$ for the quotient mapping, and note that if $M$ is a Levi factor, then $\pi_{\mid M}$ is an isomorphism of algebraic groups (see (4.3.1) below). The following example shows that a linear algebraic group may have a subgroup $M^{\prime}$ for which $\pi_{\mid M^{\prime}}$ is a purely inseparable isogeny $M^{\prime} \rightarrow G_{\text {reduc }}$ - and in particular determines an isomorphism of "abstract" groups $M^{\prime}\left(k_{\mathrm{alg}}\right) \rightarrow G_{\text {reduc }}\left(k_{\mathrm{alg}}\right)$ for an algebraic closure $k_{\text {alg }}$ of $k$-but $M^{\prime}$ is not a Levi factor of $G$.

Let $W$ be a $d$ dimensional $k$-vector space for some $d \geq 2$ where $k$ is an algebraically closed field of characteristic $p>0$, and let $V=\operatorname{Sym}^{p} W$ be the $p$-th symmetric power of $W$. Consider the subspace $W^{[1]}$ of $V$ consisting of the $p$-th powers $w^{p}$ of all vectors $w \in W$. The stabilizer $P$ in $\mathrm{GL}(V)$ of the point determined by $W^{[1]}$ in the $\operatorname{Grassmann}$ variety $\mathrm{Gr}_{d}(V)$ is a (maximal) parabolic subgroup of GL $(V)$.

Choose any linear complement $W^{\prime}$ to $W^{[1]}$ in $V$ and write $M^{\prime}$ for the reductive subgroup of $P$ generated by the image of $\mathrm{GL}(W)$ under its representation on $V$ together with the subgroup $\mathrm{GL}\left(W^{\prime}\right)$ acting as the identity on $W^{[1]}$.

(3.3.2). Write $P_{\text {reduc }}$ for the reductive quotient of $P$. Then $\pi_{\mid M^{\prime}}: M^{\prime} \rightarrow P_{\text {reduc }}$ is a purely inseparable isogeny. However, $M^{\prime}$ is not a Levi factor of $P$.

Sketch. Since $M^{\prime}(k) \cap R_{u} P(k)$ is trivial, the group of points $M^{\prime}(k)$ maps isomorphically to the group of $k$-points of the reductive quotient

$$
\left(P / R_{u} P\right)(k) \simeq \mathrm{GL}\left(W^{[1]}\right)(k) \times \mathrm{GL}\left(V / W^{[1]}\right)(k) .
$$

Thus indeed $\pi_{\mid M^{\prime}}$ is a purely inseparable isogeny.

Viewing $V$ as a representation for $\operatorname{GL}(W)$, the subspace $W^{[1]}$ is $\mathrm{GL}(W)$-invariant; the reader may verify that the exact sequence of $\mathrm{GL}(W)$-modules

$$
0 \rightarrow W^{[1]} \rightarrow V \rightarrow V / W^{[1]} \rightarrow 0
$$

is not split exact. In fact, as a representation for $\mathrm{GL}(W), V$ identifies with the standard GL $(W)$ module $H^{0}\left(\mathbf{P}(W), \mathscr{L}^{\otimes p}\right)$ of global sections of the line bundle $\mathscr{L}^{\otimes p}$ on the projective space $\mathbf{P}(W)$, where $\mathscr{L}$ is the $\mathrm{GL}(W)$-linearized line bundle whose global sections $H^{0}(\mathbf{P}(W), \mathscr{L})$ afford the natural GL $(W)$-module $W$; standard modules for GL $(W)$ are known to be indecomposable with simple socle [Ja 03, II.2.3]; in this case $\operatorname{soc}_{\mathrm{GL}(W)}(V)=W^{[1]}$ is the first Frobenius twist of the natural module $W$.

The "standard" Levi factor $M=\mathrm{GL}\left(W^{[1]}\right) \times \mathrm{GL}\left(W^{\prime}\right)$ of $P$ leaves invariant a linear complement (namely $W^{\prime}$ ) to $W^{[1]}$ in $V$. Since there is no $M^{\prime}$-stable complement to $W^{[1]}$ in $V, M^{\prime}$ is not conjugate to the Levi factor $M$. In particular, (3.3.1) shows that $M^{\prime}$ is not a Levi subgroup 
of $P$. (It is in fact quite easy to check that $d \pi_{\mid M^{\prime}}$ is not an isomorphism - the kernel of this map coincides with the Lie algebra of the image of $\mathrm{GL}(W)$.)

\section{COHOMOLOGY AND LEVI FACTORS}

4.1. The Hochschild complex. Let $G$ be a linear algebraic group over $k$. The functor $V \mapsto$ $H^{\bullet}(G, V)$ which assigns to $V$ the cohomology groups $H^{n}(G, V)$ for $n \geq 0$ is the derived functor of the fixed point functor $V \mapsto V^{G}$; cf. [Ja 03, §I.4].

Fix a representation $V$ of $G$.

(4.1.1). For any commutative $k$-algebra $\Lambda$ and any $n \geq 0$, the natural mapping

$$
H^{n}(G, V) \otimes_{k} \Lambda \stackrel{\sim}{\rightarrow} H^{n}\left(G / \Lambda, V \otimes_{k} \Lambda\right)
$$

is an isomorphism.

Proof. Since $k$ is a field, $V$ is flat over $k$ and the assertion follows from [Ja 03, Prop. I.4.18].

The cohomology $H^{\bullet}(G, V)$ can be computed using the Hochschild complex $C^{\bullet}(G, V)$; cf. [Ja 03, I.4.14]. Write $C^{\bullet}=C^{\bullet}(G, V)$. Then $C^{0}=V$, and $C^{n}=k[G]^{\otimes n} \otimes_{k} V$ for $n \geq 1$. When $V$ is finite dimensional, $V$ may be viewed as an algebraic variety over $k$ and then $C^{n}$ is the collection of regular functions $\prod^{n} G \rightarrow V$. The boundary mappings $\partial^{n}=\partial_{V}^{n}: C^{n} \rightarrow C^{n+1}$ are described in [Ja 03, I.4.14]

(4.1.2). [Ja 03, Prop I.4.16] The group cohomology $H^{\bullet}(G, V)$ is equal to the cohomology of the complex $\left(C^{\bullet}(G, V), \partial^{\bullet}\right)$.

In some arguments to follow, we shall need slightly more than the Hochschild complex itself. Following the proof of [Ja 03, Prop I.4.16] one finds:

(4.1.3). There is an injective resolution $V \rightarrow I^{\bullet}(G, V)$ of $V$ as $G$-module such that

$$
C^{\bullet}(G, V)=\left(I^{\bullet}(G, V)\right)^{G} .
$$

Proof. Indeed, as in [Ja 03, §I.4.15] one takes

$$
I^{\bullet}(G, V)=C^{\bullet}(G, k[G]) \otimes V_{\text {tr }}
$$

where $V_{\text {tr }}$ denotes $V$ with trivial $G$-action. The $G$-module structure on

$$
I^{n}(G, V)=k[G]^{\otimes(n+1)} \otimes V_{\mathrm{tr}}
$$

is given by the regular representation of $G$ on the first tensor factor; $G$ acts trivially on all other tensor factors. The boundary mapping $I^{n}(G, V) \rightarrow I^{n+1}(G, V)$ is simply $\partial_{k[G]} \otimes 1_{V}$.

Now compose the natural mapping $(v \mapsto 1 \otimes v): V \rightarrow k[G] \otimes V$ with the $G$-isomorphism

$$
k[G] \otimes V \stackrel{\sim}{\rightarrow} k[G] \otimes V_{\text {tr }}
$$

of [Ja 03, I.3.7(3)] in order to find the required G-module mapping $V \rightarrow I^{0}(G, V)$.

It is proved in [Ja 03, §1.4.15] that $0 \rightarrow V \rightarrow I^{\bullet}(G, V)$ is exact, and it is clear by construction that the complex $C^{\bullet}(G, V)$ identifies with $\left(I^{\bullet}(G, V)\right)^{G}$.

4.2. Parabolic subgroups. If $H \subset G$ is a (closed) subgroup, and if $V$ is a representation of $G$, then the co-morphism $j^{*}: k[G] \rightarrow k[H]$ to the inclusion $j: H \rightarrow G$ yields a chain mapping

$$
\rho: C^{\bullet}(G, V) \rightarrow C^{\bullet}(H, V)
$$

by the rule $\rho^{n}=\left(j^{*}\right)^{\otimes n} \otimes 1: k[G]^{\otimes n} \otimes V \rightarrow k[H]^{\otimes n} \otimes V$. In particular, if $V$ is finite dimensional and $f \in C^{n}(G, V)$ is viewed as a regular function $f: G^{n} \rightarrow V$, then $\rho(f)$ is just the restriction $f_{\mid H^{n}}: H^{n} \rightarrow V$.

Moreover, $\widetilde{\rho}=\rho \otimes 1_{V_{\text {tr }}}$ determines a chain mapping

$$
\tilde{\rho}: I^{\bullet}(G, V)=C^{\bullet}(G, V) \otimes_{k} V_{\text {tr }} \rightarrow I^{\bullet}(H, V)=C^{\bullet}(H, V) \otimes_{k} V_{\text {tr }} .
$$


We will be interested in these chain maps in the case where $H=P$ is a parabolic subgroup of a reductive group.

Proposition. Let $G$ be a reductive algebraic group over $k$, let $V$ be a representation of $G$, and let $P$ be a parabolic subgroup of $G$. Then $\rho$ induces an isomorphism $H^{i}(G, V) \stackrel{\sim}{\rightarrow} H^{i}(P, V)$ for all $i \geq 0$.

Proof. Using Kempf's vanishing theorem, it is proved in [Ja 03, II.4.7] that

$$
H^{i}(G, W) \simeq H^{i}(P, W) \text { for any representation } W \text { of } G \text {. }
$$

Note that $H^{0}(G, W)=W^{G} \subset W^{P}=H^{0}(P, W)$, so that if $W$ is finite dimensional it is immediate for dimension reasons that $W^{G}=W^{P}$. Since any $G$-representation is locally finite [Ja 03, I.2.13], the equality $W^{G}=W^{P}$ in fact holds for all G-representations $W$.

For $m \geq 0$ and $i>0$, note that $H^{i}\left(G, I^{m}(G, V)\right)=0$ since $I^{m}(G, V)$ is injective for $G$. But then $H^{i}\left(P, I^{m}(G, V)\right)=0$ by the result just cited, so the resolution $I^{\bullet}(G, V)$ of $V$ is acyclic for the functor $(-)^{P}$. It follows that $H^{\bullet}(P, V)$ may be computed as the cohomology of the complex $\left(I^{\bullet}(G, V)\right)^{P}$.

Now, the chain map $\widetilde{\rho}: I^{\bullet}(G, V) \rightarrow I^{\bullet}(P, V)$ is a quasi-isomorphism; thus [We 94, Corollary 5.7.7, 5.7.9] shows that $\widetilde{\rho}$ induces an isomorphism on hypercohomology

$$
\mathbb{H}^{\bullet}\left(P, I^{\bullet}(G, V)\right) \rightarrow \mathbb{H}^{\bullet}\left(P, I^{\bullet}(P, V)\right) .
$$

Since $I^{\bullet}(G, V)$ and $I^{\bullet}(P, V)$ are $(-)^{P}$-acyclic, the result just cited also shows that the hypercohomology groups coincide respectively with the cohomology of the complexes $I^{\bullet}(G, V)^{P}$ and $I^{\bullet}(P, V)^{P}$; this implies that

$$
\rho: C^{\bullet}(G, M)=\left(I^{\bullet}(G, V)\right)^{G}=\left(I^{\bullet}(G, V)\right)^{P} \rightarrow C^{\bullet}(P, M)=\left(I^{\bullet}(P, V)^{P}\right.
$$

is a quasi-isomorphism, as required.

(4.2.1). Let $P$ be a parabolic subgroup of $G$ and let $V$ be a linear representation of $G$. Then the restriction mapping $\left(h \mapsto h_{\mid P}\right): Z^{1}(G, V) \rightarrow Z^{1}(P, V)$ is surjective, where $Z^{1}(G, V) \subset C^{1}(G, V)$ is the group of cocycles.

Proof. Indeed, if $f \in Z^{1}(P, V)$, the Proposition shows that there is some $h \in Z^{1}(G, V)$ for which $[f]=\left[h_{\mid P}\right]$ in $H^{1}(P, V)$. Thus there is some $v \in V=C^{0}(P, V)$ for which

$$
f=h_{\mid P}+\partial_{P}(v)
$$

where $\partial_{P}(v): P \rightarrow V$ given by $x \mapsto x v-v$. Now form the 1-cocycle $h_{1}=h+\partial_{G}(v) \in$ $Z^{1}(G, V)$ where $\partial_{G}(v): G \rightarrow V$ is given by $g \mapsto g v-v$. Then evidently $h_{1 \mid P}=f$.

4.3. Group extensions. When $V$ is a finite dimensional vector space (say, underlying a module for an algebraic group), we will sometimes view $V$ as a vector group as in $\$ 2.2$ in other words, we view $V$ as the linear algebraic group over $k$ whose functor of points is given by the rule $V(\Lambda)=V \otimes_{k} \Lambda$ for each commutative $k$-algebra $\Lambda$

Let $G$ be a linear algebraic group over $k$, and let $V$ be a finite dimensional $G$-module. By an extension of $G$ by $V$, we mean a strictly exact sequence of algebraic groups (see $\$ 1.3$ for the terminology)

$$
\text { (*) } \quad 0 \rightarrow V \stackrel{i}{\rightarrow} H \stackrel{\pi}{\rightarrow} G \rightarrow 1 .
$$

We occasionally abuse terminology and refer to the extension $(*)$ simply as $H$, but the reader should be aware that $i$ and $\pi$ are "part of the data" of an extension of $G$ by $V$.

(4.3.1). Consider an extension $H$ of $G$ by $V$ given by a sequence $(*)$. Let $C$ be a closed subgroup of $H$. The following conditions are equivalent:

(a) Multiplication is an isomorphism $\mu: C \ltimes V \rightarrow H$.

(b) $\pi_{\mid C}: C \rightarrow G$ is an isomorphism of algebraic groups. 
Proof. Given (a), the map $\pi \circ \mu: C \ltimes V \rightarrow G$ is a separable surjection with kernel $\mu^{-1}(V)=V$, so that $\pi_{\mid C}=(\pi \circ \mu)_{\mid C}$ is an isomorphism; i.e. (b) holds.

Given (b), set $\sigma=i \circ\left(\pi_{\mid C}\right)^{-1}$, where $i$ is the inclusion $i: C \rightarrow H$. Then the map $H \rightarrow C \ltimes V$ given by $h \mapsto\left(\sigma \circ \pi(h),(\sigma \circ \pi(h))^{-1} h\right)$ inverts $\mu$ so that (a) holds.

A subgroup $C$ as in (4.3.1) is a complement to $V$ in $H$. We say that an extension $(*)$ of $G$ by $V$ is split if there is a complement to $V$ in $H$.

4.4. Cohomology and group extensions. Let $V$ be a representation of the linear algebraic group $G$. We relate in this section the cohomology group $H^{2}(G, V)$ and the extensions of $G$ by the vector group $V$ in the sense of 4.3 .

Consider an extension $H$ of $G$ by $V$ as in $(*)$ of 4.3 . Since the vector group $V$ is $k$-split unipotent, Proposition 2.2 shows that there is a regular function $s: G \rightarrow H$ which is a section to $\pi$. Consider now the regular mapping $\alpha_{H}: G \times G \rightarrow V$ defined by

$$
s(g) s\left(g^{\prime}\right)=i\left(\alpha_{H}\left(g, g^{\prime}\right)\right) s\left(g g^{\prime}\right)
$$

for all $g, g^{\prime} \in G(\Lambda)$ and all commutative $k$-algebras $\Lambda$.

On the other hand, given a cohomology class $[\alpha] \in H^{2}(G, V)$ represented by a 2-cocycle $\alpha: G \times G \rightarrow V$, form

$$
E_{\alpha}=G \times V
$$

with the operation $E_{\alpha} \times E_{\alpha} \rightarrow E_{\alpha}$ given by

$$
(g, v) \cdot\left(g^{\prime}, v^{\prime}\right)=\left(g g^{\prime}, v+v^{\prime}+\alpha\left(g, g^{\prime}\right)\right) .
$$

Let $i: V \rightarrow E_{\alpha}$ be the inclusion of the left-hand factor, and let $\pi: E_{\alpha} \rightarrow G$ be the projection on the right-hand factor.

Proposition. (a) For any extension $H$ of $G$ by $V$, we have $\alpha_{H} \in Z^{2}(G, V) \subset C^{2}(G, V)$, and the class $\left[\alpha_{H}\right]$ of $\alpha_{H}$ in $H^{2}(G, V)$ is independent of choices.

(b) The extension $H$ of $G$ by $V$ is split if and only if $\left[\alpha_{H}\right]=0$ in $H^{2}(G, V)$.

(c) For a 2-cocycle $\alpha \in Z^{2}(G, V)$, the indicated operation makes the variety $E=E_{\alpha}$ into a linear algebraic group which forms an extension of $G$ by $V$. Moreover, $\left[\alpha_{E}\right]=[\alpha]$ in $H^{2}(G, V)$.

Proof. [DeGa 70, II $\S 3.2 .3]$

Remark. (a) Let $G$ be a split, semisimple group over a perfect field $k$. Recall that by (3.2.1), there is a non-trivial extension of a $G$ by the Frobenius twist $\mathfrak{g}^{[1]}$ of its Lie algebra, viewed as a vector group with $G$-action. Thus, the Proposition shows that $H^{2}\left(G_{/ k}, \mathfrak{g}^{[1]}\right) \neq 0$ for any split semisimple algebraic group $G$ over $k$.

(b) Using the non-vanishing of a certain second cohomology group, we give in $\$ 7.1$ an example of a linear algebraic group with no Levi decomposition

4.5. Conjugacy of complements. Let $V$ be a representation for the linear algebraic group $G$, and form the semidirect product $H=G \ltimes V$ which we view as the split extension of $G$ by $V$. Write $\pi: H \rightarrow G$ for the quotient homomorphism $(g, v) \mapsto g$, write $\sigma_{0}: G \rightarrow H$ for the inclusion $g \mapsto(g, 0)$ of $G$ in $H$, write $i: V \rightarrow H$ for the inclusion $v \mapsto(1, v)$ of $V$ in $H$, and write $\psi: H=G \ltimes V \rightarrow V$ for the regular map of varieties $(g, v) \mapsto v$; i.e. $\psi$ is given by projection on the $V$ factor.

Let $\Sigma=\{\sigma: G \rightarrow H \mid \sigma$ is both a section to $\pi$ and a homomorphism of algebraic groups $\}$.

(4.5.1). The assignment $\sigma \mapsto \psi \circ \sigma$ determines a bijection $\Sigma \rightarrow Z^{1}(G, V)$ where $Z^{1}(G, V)$ is the set of co-cycles in $C^{1}(G, V)$. 
Proof. Given $\sigma \in \Sigma$, one easily checks that $f=\psi \circ \sigma \in Z^{1}(G, V)$. Conversely, given $f \in$ $Z^{1}(G, V)$ we may form the section $\sigma_{f}: G \rightarrow H=G \ltimes V$ given by the rule

$$
g \mapsto(g, f(g)) ;
$$

the co-cycle condition implies that $\sigma$ is a homomorphism of algebraic groups.

It is clear that the assignments $\sigma \mapsto \psi \circ \sigma$ and $f \mapsto \sigma_{f}$ are inverse to one another.

(4.5.2). Let $\sigma \in \Sigma$, and let $f=\psi \circ \sigma \in Z^{1}(G, V)$ be the corresponding co-cycle as in (4.5.1) The following are equivalent:

(a) $[f]=0$ in $H^{1}(G, V)$.

(b) The sections $\sigma, \sigma_{0} \in \Sigma$ are conjugate by an element $i(v) \in H(k)$ for some $v \in V=V(k)$

(c) The sections $\sigma, \sigma_{0} \in \Sigma$ are conjugate by an element of $H\left(k_{\mathrm{alg}}\right)$ where $k_{\mathrm{alg}}$ is an algebraic closure of $k$.

Proof. By definition, the condition $[f]=0$ in $H^{1}(G, V)$ is equivalent to the condition $f=\partial v$ for some $v \in V=C^{0}(G, V)$ which simply means that $f: G \rightarrow V$ is given by the rule $f(g)=g v-v$. Now, for each commutative $k$-algebra $\Lambda$ and each $g \in G(\Lambda)$ we have

$$
i(v) \cdot \sigma_{0}(g) \cdot i(-v)=(1, v)(g, 0)(1,-v)=(g, g v-v)=(g, f(g))=\sigma(g) ;
$$

this proves the equivalence of (a) and (b).

Evidently (b) implies (c). If (c) holds, say $\sigma=\operatorname{Int}(h) \sigma_{0}$ for $h \in H\left(k_{\text {alg }}\right)$, we may write $h=(g, v)$ for $v \in V\left(k_{\mathrm{alg}}\right)$ and $g \in G\left(k_{\mathrm{alg}}\right)$. Since $1_{G}=\pi \circ \sigma=\pi \circ \sigma_{0}$, evidently $g$ is central so that in fact $\sigma=\operatorname{Int}(i(v)) \sigma_{0}$. But then $[f]=0$ in $H^{1}\left(G_{/ k_{\mathrm{alg}}}, V_{/ k_{\mathrm{alg}}}\right)$ by the equivalence of (b) and (a) for $G_{/ k_{\text {alg }}}$, whence (a) by application of (4.1.1).

\section{SOME COHOMOLOGICAL CRITERIA FOR EXISTENCE AND CONJUGACY OF LEVI FACTORS}

Throughout this section, $G$ will denote a connected linear algebraic group. We suppose throughout $\$$ that condition $(L)$ of $\$ 2.3$ holds for $G$.

We fix a linearizable splitting sequence $1=R_{n} \subset R_{n-1} \subset \cdots \subset R_{0}=R$ for $R$ and write $V_{i}$ for the linear $G_{\text {reduc }}$-representations for which there are $G_{\text {reduc }}$-equivariant isomorphisms $V_{i} \simeq R_{i} / R_{i-1}$.

\subsection{A cohomological criteria for conjugacy of Levi factors.}

Theorem. Suppose that $G$ has a Levi factor, and suppose that

$$
H^{1}\left(G_{\text {reduc }}, V_{i}\right)=0 \text { for } 0 \leq i \leq n-1 .
$$

Then any two Levi factors $L, L^{\prime}$ of $G$ are conjugate by an element of $R(k)$.

Proof. Proceed by induction on the length $n$ of a linearizable splitting sequence for $R$. In case $n=1$, the result follows from (4.5.2)

Now let $n>1$ and suppose the result holds for groups having linearizable splitting sequences of length $<n$. Let $L, L^{\prime} \subset G$ be Levi factors.

The images $\bar{L}, \overline{L^{\prime}}$ of the Levi factors of $G$ in $G / R_{1}$ are Levi factor of $G / R_{1}$. Identifying $V_{0}$ and $R / R_{1}$, we view $G / R_{1}$ as an extension

$$
0 \rightarrow V_{0} \rightarrow G / R_{1} \rightarrow G_{\text {reduc }} \rightarrow 1
$$

and it follows from (4.5.2) (i.e. the case $n=1)$ that there is $v \in V_{0}$ such that $\bar{L}=\operatorname{Int}(i(v)) \overline{L^{\prime}}$. Now, the morphism $R(k) \rightarrow\left(R / R_{1}\right)(k)=V_{0}$ is surjective by Proposition 2.2 Choosing an $x \in R(k)$ which maps to $v \in V_{0}$ and replacing $L^{\prime}$ by $\operatorname{Int}(x) L^{\prime}$ we may suppose that $\bar{L}=\overline{L^{\prime}}$.

Let $\pi: G \rightarrow G / R_{1}$ be the quotient mapping, and let $M=\pi^{-1}(\pi(L))=\pi^{-1}(\bar{L})$. Then $M$ is an extension

$$
1 \rightarrow R_{1} \rightarrow M \rightarrow G_{\text {reduc }} \rightarrow 1 ;
$$


since $\bar{L}=\overline{L^{\prime}}$, we have $L, L^{\prime} \subset M$ so that both $L$ and $L^{\prime}$ are Levi factors of $M$. Since a splitting sequence for the unipotent radical $R_{1}$ of $M$ has length $n-1$, we may apply induction to learn that $L$ and $L^{\prime}$ are conjugate by an element of $R_{1}(k)$.

\subsection{A cohomological criteria for the existence of a Levi factor.}

Theorem. Suppose that

$$
H^{2}\left(G_{\text {reduc }}, V_{i}\right)=0 \text { for } \quad 0 \leq i \leq n-1 \text {. }
$$

Then $G$ has a Levi factor.

Sketch: One proves the result by induction on the length $n$ of a linearizable splitting sequence for $R$; the result in case $n=1$ follows from Proposition 4.4. The induction step is similar to in fact, easier than - that given in the proof of Theorem 5.1; details are left to the reader.

5.3. Dimensional criteria for existence and conjugacy of Levi factors. Let $H$ be a reductive algebraic group over $k$. A result of Jantzen permits some control on the cohomology of $\mathrm{H}$ modules whose dimension is small relative to the size of the characteristic of $k$, as follows:

(5.3.1) (Jantzen [Ja 97]). If $V$ is an H-module with $\operatorname{dim} V \leq p$, then $V$ is completely reducible.

(5.3.2). If $V$ is a $H$-module with $\operatorname{dim} V<p$, then $H^{1}(H, V)=0$.

Proof. Using[(4.1.1) we may as well suppose that $k$ is algebraic closed. Suppose that

$$
0 \neq H^{1}(H, V)=\operatorname{Ext}_{H}^{1}(k, V) .
$$

Then a non-zero cohomology class determines a non-split extension of $G$-modules

$$
0 \rightarrow V \rightarrow E \rightarrow k \rightarrow 0
$$

in particular, the $H$-module $E$ is not completely reducible. Now (5.3.1) implies that $\operatorname{dim} E>p$ so that $\operatorname{dim} V \geq p$.

(5.3.3). Let $V$ be an H-module with $\operatorname{dim} V \leq p$ and suppose that there are dominant weights $\mu_{1}, \ldots, \mu_{n}$ such that the character of $V$ satisfies ch $V=\sum_{j} \chi\left(\mu_{j}\right)$. Then $H^{i}(H, V)=0$ for $i \geq 1$.

Proof. The hypotheses imply that $\operatorname{dim} H^{0}\left(\mu_{j}\right) \leq p$ for all $j$. Now Janzten's result (5.3.1) implies that $V$ together with each $H^{0}\left(\mu_{j}\right)$ is completely reducible. In particular, $H^{0}\left(\mu_{j}\right)=L\left(\mu_{j}\right)$ is simple. Thus (2.4.3) implies that the composition factors of $V$ are precisely the $L\left(\mu_{j}\right)$. In turn, the complete reducibility of $V$ shows that $V$ is the direct sum $V=\oplus_{j} L\left(\mu_{j}\right)=$ $\oplus_{j} H^{0}\left(\mu_{j}\right)$, and the result now follows from (2.4.2).

Theorem. Suppose that condition ( $L$ ) holds for $G$ and write $p$ for the characteristic of $k$. Let $1=R_{n} \subset$ $R_{n-1} \subset \cdots \subset R_{0}=R$ be a linearizable splitting sequence for $R$, and suppose that $R_{i} / R_{i-1} \simeq V_{i}$ for G-modules $V_{i}$.

(a) Suppose that $\operatorname{dim} R<p$. If $G$ has a Levi factor, then any two Levi factors are conjugate by an element of $R(k)$.

(b) Suppose that $\operatorname{dim} R \leq p$. If there are dominant weights $\mu_{1}, \ldots, \mu_{m}$ for $G_{\text {reduc }}$ such that

$$
\sum_{j=0}^{n-1} \operatorname{ch} V_{j}=\sum_{i=1}^{m} \chi\left(\mu_{i}\right)
$$

then $G$ has a Levi factor.

Proof. Assertion (a) is a consequence of (5.3.2) and Theorem 5.1, and assertion (b) is a consequence of (5.3.3) and Theorem5.2. 
Remark. Suppose that the reductive group $H$ has absolutely quasi-simple derived group and semisimple rank $r$. The main result of [Mc 98] shows that any $H$-representation with $\operatorname{dim} V \leq$ $r p$ is completely reducible; this leads to obvious analogues of (5.3.2) and (5.3.3). If $H$ is isomorphic to the reductive quotient of $G$, the conclusion of the preceding Theorem remains valid after replacing in (a) the condition " $\operatorname{dim} R<p$ " by the condition " $\operatorname{dim} R<r p$ " and in (b) the condition " $\operatorname{dim} R \leq p$ " by the condition " $\operatorname{dim} R \leq r p$ ".

5.4. Another criteria for the existence of a Levi factor. Suppose in this section that $k$ is separably closed. Then the reductive quotient $G_{\text {reduc }}=G / R$ is split and in particular has a Borel subgroup over $k$.

Fix a Borel subgroup $B \subset G_{\text {reduc }}=G / R$, and write $\widetilde{B}=\pi^{-1}(B) \subset G$ where $\pi: G \rightarrow G_{\text {reduc }}$ is the quotient mapping. We may view $\widetilde{B}$ as an extension

$$
1 \rightarrow R \rightarrow \widetilde{B} \rightarrow B \rightarrow 1 \text {. }
$$

We assume that (5.4.a) is split, and we fix a homomorphism $\sigma: B \rightarrow \widetilde{B}$ which is a section to this extension. Write $B_{1}$ for the image of $\sigma$. Under these assumptions, we find:

(5.4.1). G has a Levi factor containing $B_{1}$.

Proof. Recall that we have fixed a linearizable splitting sequence $1=R_{n} \subset R_{n-1} \subset \cdots \subset R_{0}=$ $R$ for $R$; for each $i$, the vector group $R_{i} / R_{i+1}$ is $G_{\text {reduc }}$-equivariantly isomorphic to the linear representation $V_{i}$ of $G_{\text {reduc }}$.

We prove the result by induction on $n$. First suppose that $n=1$; then $R \simeq V_{0}=V$ is a linearizable vector group. Viewing $G$ as an extension of $G_{\text {reduc }}$ by $V$, we find a 2-cocycle $\alpha=\alpha_{G} \in Z^{2}(G, V)$ as in $\$ 4.4$ The restriction to $B$ (more precisely: to $B \times B$ ) of $\alpha$ is the 2-cocycle determined by the extension (5.4.a); thus by hypothesis and by Proposition 4.4, we have $\left[\alpha_{\mid B \times B}\right]=0$ in $H^{2}(B, V)$. But then $[\alpha]=0$ in $H^{2}(G, V)$ by Proposition 4.2, so another application of Proposition 4.4 shows that the extension

$$
0 \rightarrow V \rightarrow G \rightarrow G_{\text {reduc }} \rightarrow 1
$$

is split. Let $\tau: G_{\text {reduc }} \rightarrow G$ be a homomorphism which is a section to this extension. Restricting $\tau$ to the Borel group $B$ gives a section $\tau_{\mid B}: B \rightarrow \widetilde{B}$. According to (4.5.1), there is $f \in Z^{1}(B, V)$ for which

$$
\sigma(b)=\tau(b) \cdot i(f(b)) \quad \text { for each commutative } k \text {-algebra } \Lambda \text { and each } b \in B(\Lambda) \text {. }
$$

According to (4.2.1) we may find $h \in Z^{1}(G, V)$ such that $f=h_{\mid B}$. Now the image of the section $G_{\text {reduc }} \rightarrow G$ given by $g \mapsto \tau(g) \cdot i(h(g))$ is a Levi factor of $G$ containing $B_{1}$, as required.

Now suppose $n>1$ and that the conclusion of the Theorem is known for groups having linearizable splitting sequences of length $<n$. Let $\overline{B_{1}}$ be the image of $B_{1}$ in $G / R_{1}$. Then $\overline{B_{1}}$ is a complement to $R / R_{1}$ in $\widetilde{B} / R_{1}$, so that the extension

$$
0 \rightarrow R / R_{1} \rightarrow \widetilde{B} / R_{1} \rightarrow B \rightarrow 1
$$

is split; thus by the case $n=1$, we may find a Levi factor $\bar{M} \subset G / R_{1}$ containing $\overline{B_{1}}$.

Write $\pi: G \rightarrow G / R_{1}$ for the quotient mapping, and consider

$$
\widetilde{B_{1}}=\pi^{-1}\left(\overline{B_{1}}\right) \subset M=\pi^{-1}(\bar{M}) .
$$

After identifying $\bar{M}$ with $G_{\text {reduc }}$, we may view $M$ and $\widetilde{B_{1}}$ as extensions

$$
1 \rightarrow R_{1} \rightarrow M \rightarrow G_{\text {reduc }} \rightarrow 1 \text { and } 1 \rightarrow R_{1} \rightarrow \widetilde{B_{1}} \rightarrow B \rightarrow 1 .
$$

Moreover, $B_{1} \subset \widetilde{B_{1}}$ so that $\sigma: B \rightarrow \widetilde{B_{1}}$ is a section to the second extension. Since the unipotent radical $R_{1}$ of the linear algebraic group $M$ has a linearizable splitting sequence of length $n-1$, induction gives a Levi factor $N$ of $M$ containing $B_{1}$, and evidently $N$ is the desired Levi factor of $G$. 
5.5. Existence of a unique Levi factor containing a fixed maximal torus. We prove in this section the existence result for Levi factors that is applied below in 86

Fix a maximal torus $T$ of $G$, and let $T_{\text {reduc }}$ denote the image of $T$ under $\pi$; then $T_{\text {reduc }}$ is a maximal torus of $G_{\text {reduc }}$.

(5.5.1). Suppose that there is a unique Levi factor $M$ of $G_{/ k_{\text {sep }}}$ containing $T_{/ k_{\text {sep }}}$. Then $M$ is defined over $k$ and is the unique Levi factor of $G$ containing $T$.

Proof. Write $\Gamma$ for the Galois group of $k_{\text {sep }} / k$. Since $T$ is defined over $k$, it is stable by the action of $\Gamma$. The unicity of $M$ then shows that $M$ is $\Gamma$ stable; thus $M$ is defined over $k$ by Galois descent [Sp 98, 11.2.8].

Theorem. Let $k$ be separably closed, fix a Borel subgroup $B \subset G_{\text {reduc }}$ containing $T_{\text {reduc, }}$ and write $\widetilde{B}=\pi^{-1}(B)$ where $\pi: G \rightarrow G_{\text {reduc }}=G / R$ is the quotient mapping. Assume that there is a unique

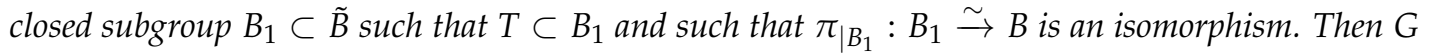
has a unique Levi factor containing $T$.

Proof. Since $k$ is assumed to be separably closed and since the extension $1 \rightarrow R \rightarrow \widetilde{B} \rightarrow B \rightarrow 1$ is split by hypothesis, we may apply (5.4.1); it gives a Levi factor $M \subset G$ containing $B_{1}$ and in particular containing $T$. It only remains to argue the uniqueness.

Before proceeding further, we observe first that if $B^{\prime}$ is any Borel subgroup of $G_{\text {reduc }}$ (over $k$ ) containing $T_{\text {reduc }}$, the hypothesis shows: $(*)$ there is a unique closed $B_{1}^{\prime} \subset \pi^{-1}\left(B^{\prime}\right)$ such that $\pi_{\mid B_{1}^{\prime}}: B_{1}^{\prime} \stackrel{\sim}{\rightarrow} B$ is an isomorphism. Indeed, since $k$ is separably closed, [Bo 91, Theorem V.20.9] shows that any two Borel subgroups of $G_{\text {reduc }}$ are conjugate by an element of $G_{\text {reduc }}(k)$, and the claim follows since the mapping $G(k) \rightarrow G_{\text {reduc }}(k)$ is surjective by Proposition 2.2 .

Suppose now that $M, M^{\prime}$ are two Levi factors of $G$ each containing $T$. The assumption shows that both $M$ and $M^{\prime}$ contain $B_{1}$. Choose in the reductive group $M$, resp. $M^{\prime}$, a Borel subgroup $C$, resp. $C^{\prime}$, containing $T$ opposite to $B_{1}$

Then $\pi(C)$ and $\pi\left(C^{\prime}\right)$ are Borel subgroups of $G_{\text {reduc }}$ containing $T$ and opposite to $B$. Hence $\pi(C)$ and $\pi\left(C^{\prime}\right)$ are equal by [Bo 91, Prop. IV.14.21(i)]. But then $C=C^{\prime}$ by the observation $(*)$. Then $M$ and $M^{\prime}$ each contain the variety $C B_{1}=C^{\prime} B_{1}$ as an open, dense subset [Bo 91, Prop. 1V.14.21(iii)], hence $M=M^{\prime}$ as required.

\section{PARAHORIC GROUP SCHEMES}

Let $\mathscr{A}$ be a Henselian discrete valuation ring (DVR) with maximal ideal $\mathfrak{m}=\mathscr{C} \mathscr{A}$. Henselian means that $\mathscr{A}$ satisfies the conclusion of Hensel's Lemma; for example, the DVR $\mathscr{A}$ is Henselian if it is complete in its $\mathfrak{m}$-adic topology. We write $K$ for the field of fractions of $\mathscr{A}$ and $k$ for the residue field of $\mathscr{A}$; we assume throughout $\sqrt{6}$ that the residue field $k$ is perfect.

We denote by $G$ a connected and reductive group over the field $K$. We will be interested in certain algebraic groups over $k$ which arise in the study of this group $G$. These are the special fibers $\mathcal{P}_{/ k}$ of certain smooth $\mathscr{A}$-group schemes $\mathcal{P}$ associated with $G$ known as parahoric group schemes.

As hinted at in the introduction, these parahoric group schemes are related to the stabilizers in $G(K)$ of certain subsets of the affine building $\mathscr{I}$ of $G$. The reader is referred to [Ti 77], [BrTi 72] and [BrTi 84] for more on these matters.

For our purposes in this paper, we do not need to discuss in detail this affine building. When $G$ is split over $K$, we will just describe the parahoric group schemes associated to the "standard alcove" in a fixed apartment; up to isomorphism of $\mathscr{A}$-group schemes, these account for all parahoric group schemes associated with $G$. When $G$ splits over an unramified extension $L / K$, every parahoric group scheme for $G$ is obtained by "étale descent" from one for the split group $G / L$.

These descriptions will permit us to give a proof of Theorem A from $\$ 1$ 
6.1. An apartment in case $G$ is split over $K$. Suppose in 8.1 that $G$ is split over $K$ and that $T$ is a maximal split $K$-torus of $G$.

In order to describe the parahoric group schemes associated with $G$, we must first describe a part of the Bruhat-Tits building $\mathscr{I}$ associated with $G$, namely, the apartment $A$ corresponding to $T$.

Write $\Phi \subset X^{*}(T)$ for the roots of $G$, and for $a \in \Phi$ write $U_{a}$ for the corresponding root subgroup of $G$. Fix a Chevalley system, i.e. a choice of K-isomorphisms $\chi_{a}: \mathbf{G}_{a} \rightarrow U_{a}$ for each $a \in \Phi$ satisfying the "usual" commutation relations; see [BrTi 84, (3.2.2)].

Now write $A=X_{*}(T) \otimes \mathbf{R}$, where $X_{*}(T)$ is the lattice of co-characters of the split torus $T$. An affine function on $A$ has the form $\phi=(\psi, r)$ where $\psi \in A^{\vee}=X^{*}(T) \otimes \mathbf{R}$ and $r \in \mathbf{R}$; thus $\phi(a)=\psi(a)+r$ for $a \in A$, and $\psi$ is the gradient of $\phi$. The affine roots $\Phi^{\text {aff }}$ of $G$ with respect to $T$ are the affine functions on $A$ of the form $\alpha=(a, \gamma)$ for $a \in \Phi$ and $\gamma \in \mathbf{Z}$.

The walls of $A$ are the subsets $\alpha^{-1}(0)$ for $\alpha \in \Phi^{\text {aff }}$; the connected components of the complement of the union of all walls are the alcoves of $A$, and the facets of these alcoves are the facets of $A$.

Write $\Phi=\bigcup_{i \in I} \Phi_{i}$ as the disjoint union of its irreducible components $\Phi_{i}$, fix for each $i$ a basis of simple roots $\Delta_{i}$ for the root system $\Phi_{i}$, and let $\tilde{\alpha}_{i} \in \Phi_{i}$ be the highest root (i.e. the dominant long root for the given choice of basis). Now write $\Delta_{i}^{0}$ for the set of affine roots

$$
\Delta_{i}^{0}=\left\{(a, 0) \mid a \in \Delta_{i}\right\} \cup\left\{\left(-\tilde{\alpha}_{i}, 1\right)\right\}
$$

and write $\Delta^{0}=\bigcup_{i \in I} \Delta_{i}^{0}$.

The fundamental alcove is the set $C=\bigcap_{\alpha \in \Delta^{0}} \alpha^{-1}((0, \infty))$; when $G$ is (quasi-) simple, $C$ is a simplex; for semisimple $G, C$ is a direct product of simplices, and in general $C$ is a direct product of simplices and a Euclidean space.

(6.1.1). The facets in the closure of the fundamental alcove $C$ are in bijection with the subsets $\Theta=$ $\cup_{i \in I} \Theta_{i} \subset \Delta^{0}$ where $\Theta_{i}$ is a non-empty subset of $\Delta_{i}^{0}$ for each $i \in I$. The facet $\mathcal{F}_{\Theta}$ determined by the subset $\Theta$ is given by

$$
\mathcal{F}_{\Theta}=\left(\bigcap_{\alpha \in \Theta} \alpha^{-1}((0, \infty))\right) \cap\left(\bigcap_{\alpha \in \Delta^{0}-\Theta} \alpha^{-1}(0)\right) .
$$

Proof. The assertion is easily reduced to the case where $\Phi$ is irreducible, and in that case it follows from [Bou 02, Corollary VI.2.3].

(6.1.2). Let $\Phi_{\Theta}$ be the set of all roots $a \in \Phi$ such that there is $\alpha \in \Phi^{\text {aff }}$ with gradient a for which $\alpha_{\mid \mathcal{F}}=0$. Then $\Phi_{\Theta}$ is a closed system of roots in $\Phi$. The Dynkin diagram of $\Phi_{\Theta}$ is obtained from the extended Dynkin diagram of $\Phi$ by deleting the nodes corresponding to $\Theta$ and all edges adjacent to those nodes.

Proof. If $a, b \in \Phi_{\Theta}$ then there are integers $\gamma_{a}, \gamma_{b}$ such that the affine roots $\left(a, \gamma_{a}\right)$ and $\left(b, \gamma_{b}\right)$ vanish on $\mathcal{F}$. Now, if $a+b \in \Phi$ then evidently $\left(a+b, \gamma_{a}+\gamma_{b}\right)$ vanishes on $\mathcal{F}$ so that $a+b \in$ $\Phi_{\Theta}$, as required. For the second assertion, see (e.g.) [PR 84, 2.22].

Remark. (a) Strictly speaking, the apartment $A$ of the affine building $\mathscr{I}$ of $G$ associated with the torus $T$, should be instead an affine space under $X_{*}(T) \otimes \mathbf{R}$; our choice of Chevalley system determines an "origin" for $A$ and permits our identification of $A$ with $X_{*}(T) \otimes \mathbf{R}$; see $\S 1$ of [Ti 77] for a nice account of these matters.

(b) If $W=N_{G}(T) / T$ denotes the Weyl group of $G$ and $W^{\text {aff }}=W \ltimes X_{*}(T)$ the affine Weyl group, then any alcove in $A$ is conjugate by $W^{\text {aff }}$ to the fundamental alcove $C$; for this reason, we focus attention on the alcove $C$. 
6.2. Parahoric group schemes in case $G$ is split over $K$. Keep the assumptions and notations of 6.1 and fix a facet $\mathcal{F}$ of the standard alcove $C$. Thus $\mathcal{F}=\mathcal{F}_{\Theta}$ for some $\Theta$ as in (6.1.1). We are going to describe the parahoric group scheme associated with the facet $\mathcal{F}$.

This parahoric group scheme is constructed from $\mathscr{A}$-group schemes attached to $T$ and the $U_{a}$. Since $T$ is a split $K$-torus, there is a canonical split $\mathscr{A}$-torus $\mathcal{T}$ with generic fiber $\mathcal{T}_{/ K}=T$ for which $X^{*}(T)=X^{*}(\mathcal{T})$; see [BrTi 84, 1.2.11].

Given $\alpha=(a, \gamma) \in \Phi^{\text {aff }}$ with gradient $a \in \Phi$, we get a subgroup $U_{\alpha} \subset U_{a}(K)$ by the rule $U_{\alpha}=\chi_{a}\left(\omega^{\gamma} \mathscr{A}\right)$ where $\chi_{a}: \mathbf{G}_{a} \rightarrow U_{a}$ is the isomorphism involved in the Chevalley system. We get moreover a smooth $\mathscr{A}$-group scheme $\mathcal{U}_{\alpha}$ with generic fiber $U_{a}$ obtained by transport of structure via $\chi_{a}$ from the $\mathscr{A}$-group scheme defined by the $\mathscr{A}$-module $\omega^{\gamma} \mathscr{A}$ as in [BrTi 84, (1.4.1)]; then $\mathcal{U}_{\alpha}(\mathscr{A})=U_{\alpha}$.

For $a \in \Phi^{+}$, let $e_{a}=0$ if the root $a$ vanishes on $\mathcal{F} \subset A$, and let $e_{a}=1$ otherwise; note in the latter case that $1>a(x)>0$ for each $x \in \mathcal{F}$. Consider the following set of affine roots:

$$
\Psi=\Psi_{\Theta}=\left\{(a, 0) \mid a \in \Phi^{+}\right\} \cup\left\{\left(-a, e_{a}\right) \mid a \in \Phi^{+}\right\}
$$

(6.2.1). $\left(\mathcal{T},\left(\mathcal{U}_{\alpha}\right)_{\alpha \in \Psi_{\Theta}}\right)$ is a schematic root datum over $\mathscr{A}$. There is a smooth $\mathscr{A}$-group scheme $\mathcal{P}$ with generic fiber $G$ such that the injections of $T$ and the $U_{a}$ in $G$ prolong to isomorphisms of $\mathcal{T}$ and the $\mathcal{U}_{a}$ onto closed $\mathscr{A}$-subschemes of $\mathcal{P}$. With the obvious definitions of the sets of affine roots $\Psi^{ \pm}$, the product mappings yield isomorphisms of $\prod_{\alpha \in \Psi^{ \pm}} \mathcal{U}_{\alpha}$ onto closed subgroup schemes $\mathcal{U}^{ \pm}$of $\mathcal{P}$, and the product mapping yields an isomorphism of $\mathcal{U}^{-} \times \mathcal{T} \times \mathcal{U}^{+}$onto an open subscheme of $\mathcal{P}$.

Proof. This follows from [BrTi 84, 3.8.1, 3.8.3, $§ 4.6$ and 4.6.26]; we only need to observe that the function $f_{\mathcal{F}}$ of loc. cit., 4.6.26 takes the value 0 on any $a \in \Phi^{+}$, while for $a \in \Phi^{+}, f_{\mathcal{F}}(-a)=0$ if $e_{a}=0$ and $0<f_{\mathcal{F}}(-a)<1$ if $e_{a}=1$.

(6.2.2) ([PR 84, 2.22] or [BrTi 84, 4.6.12]). The root system of the reductive quotient of the special fiber $\mathcal{P}_{/ k}$ is $\Phi_{\Theta}$.

(6.2.3). Fix a Borel subgroup $B$ containing $\mathcal{T}_{/ k}$ in the reductive quotient $\mathcal{P}_{/ k \text {,reduc }}$ of the special fiber of $\mathcal{P}$, and let $\tilde{B}=\pi^{-1}(B) \subset \mathcal{P}_{/ k}$ where $\pi$ is the quotient mapping. There is a unique subgroup $B_{1} \subset \tilde{B}$ such that $\mathcal{T}_{/ k} \subset B_{1}$ and such that $\pi_{\mid B_{1}}: B_{1} \rightarrow B$ is an isomorphism.

Proof. The existence of $B_{1}$ follows from (6.1.2) together with [BrTi 84, Cor. 4.6.4(ii)], since a positive system of roots in $\Phi_{\Theta}$ is quasi-closed in the terminology of loc. cit.. Uniqueness follows from op. cit. Cor. 4.6.4(i) since any $B_{1}$ must contain the root subgroup of $\mathcal{P}_{/ k}$ for each $a \in \Phi_{\Theta}^{+}$.

6.3. Condition (L) for the special fiber of a parahoric group scheme in the split case. Keep the notation and assumptions of 6.2 , and suppose in addition that the residue field $k$ of $\mathscr{A}$ is algebraically closed.

In this case, we verify that condition (L) holds for the special fiber $\mathcal{P}_{/ k}$ of the parahoric group scheme $\mathcal{P}_{\mathcal{F}}$ determined by the facet $\mathcal{F}=\mathcal{F}_{\Theta}$.

Recall that $\Phi=\cup_{i \in I} \Phi_{i}$.

(6.3.1) (see e.g. [PR 84, 2.10]). For an affine root $\alpha \in \Phi^{\text {aff, }}$, let $i \in I$ such that the gradient of $\alpha$ is in $\Phi_{i}$. Then we may write $\alpha=\sum_{\beta \in \Delta_{i}^{0}} t_{\beta} \beta$ for unique integers $t_{\beta}$, and the $t_{\beta}$ are either all non-negative or all non-positive.

We define a function $\ell_{\Theta}$ on affine roots as follows: if $\alpha \in \Phi^{\text {aff }}$ has gradient $a \in \Phi_{i} \subset \Phi$, we set

where the $t_{\alpha}$ are as in (6.3.1)

$$
\ell_{\Theta}(\alpha)=\sum_{\alpha \in \Theta_{i}} t_{\alpha}
$$


Write $\delta=(0,1)$ for the constant affine function on $A$. Then for $i \in I$, we may write $\delta=\sum_{\alpha \in \Delta_{i}^{0}} m(i)_{\alpha} \alpha$ for unique positive integers $m(i)_{\alpha}$, and we set

$$
\ell_{i}=\sum_{\alpha \in \Delta_{i}^{0}} m(i)_{\alpha} \text { for } i \in I
$$

(6.3.2). The special fiber $\mathcal{P}_{/ k}$ satisfies condition $(L)$ of 2.3

Proof. It follows from [PR 84, 2.22] that the unipotent radical $R$ of $\mathcal{P} / k$ is generated by the canonical images in $\mathcal{P}(k)$ of the groups $\mathcal{U}_{\alpha}(\mathscr{A})$ for all affine roots $\alpha$ with $\ell_{\Theta}(\alpha) \geq 1$.

Moreover, it is straightforward to verify that $R=\prod_{j} R(j)$ is the direct product of the Ginvariant subgroups $R(j)$ generated by the canonical images of those groups $\mathcal{U}_{\alpha}(\mathscr{A})$ as above for which $\alpha$ has gradient in $\Phi_{j}$. Thus it suffices to prove the assertion in the case where $\Phi=\Phi_{1}$ is irreducible, which we now suppose. Write $\ell$ for $\ell_{1}$ as in 6.3.a).

Let $0 \leq i \leq \ell-1$. Since $k$ is algebraically closed, the canonical images in $\mathcal{P}(k)$ of the groups $\mathcal{U}_{\alpha}(\mathscr{A})$ for which $\ell_{\Theta}(\alpha) \geq i+1$ generate a closed, connected subgroup $R_{i} \subset \mathcal{P} / k$.

It follows from [PR 84, 2.16] that $R_{\ell-1}=1$ and loc. cit., 2.22 shows for $0 \leq i \leq \ell-2$ that the quotient $R_{i} / R_{i+1}$ has a natural structure of a finite dimensional $k$-vector space. The usual commutator relations [Sp 98, 8.2.3] for $G$ imply that the reductive quotient of $\mathcal{P}_{/ k}$ acts linearly on each quotient, thus indeed (L) holds for $\mathcal{P}_{/ k}$.

6.4. The maximal unramified extension. Fix a separable closure $K_{\text {sep }}$ of $K$, and let $K_{\text {un }}$ denote the maximal unramified extension of $K$ in $K_{\text {sep }}$. The integral closure $\mathscr{A}$ un of $\mathscr{A}$ in $K_{\text {un }}$ is a DVR whose residue field is an algebraic closure $k_{\text {alg }}$ of the perfect field $k$.

We consider the reductive $K_{\text {un }}$-group $G / K_{\text {un }}$ obtained from $G$ by base-change. By a theorem of Lang [Ser97. II.3.3], $K_{\mathrm{un}}$ is a $C_{1}$ field. Thus, we have the following important result due to Steinberg (in case $K$ is perfect) and Borel-Springer:

(6.4.1) ([BS68]). The reductive group $G_{/ K_{\mathrm{un}}}$ is quasi-split; i.e. $G_{/ K_{\mathrm{un}}}$ has a Borel subgroup defined over $K_{\mathrm{un}}$.

When $G_{/ K_{\mathrm{un}}}$ is split over $K_{\mathrm{un}}$, we have the following result:

(6.4.2) ([BrTi 84, 5.1.12]). There is a maximal K-split torus $S$ of $G$ and a maximal torus $T$ of $G$ containing $S$ for which $T_{/} K_{\mathrm{un}}$ is $K_{\mathrm{un}}$-split.

6.5. Parahoric group schemes for $G$ and étale descent. Let $G$ be a connected, reductive group over $K$.

If $G$ is quasi-split - i.e. has a Borel subgroup over $K$ - the parahoric group schemes associated with $G$ can be described in an explicit manner analogous to our description in $\$ 6.2$ when $G$ is split; see [BrTi 84, §4].

In general, the parahoric group schemes for $G$ arise by étale descent from those of $G / K_{\mathrm{un}}$. We will describe this descent when $G_{/ K_{\mathrm{un}}}$ is split over $K_{\mathrm{un}}$. Fix $S \subset T \subset G$ as in (6.4.2). Then $T_{K_{\text {un }}}$ is a $K_{\text {un }}$-split torus of $G_{/ K_{\text {un }}}$; write $A_{\text {un }}$ for the apartment associated with $T_{\text {un }}$ as in $\$ 6.1$ The Galois group $\Sigma=\operatorname{Gal}\left(K_{\mathrm{un}} / K\right)$ acts on $A_{\mathrm{un}}$, and we have the following:

(6.5.1) (Étale descent). Let $\mathcal{F}$ be a $\Sigma$-invariant facet of $A_{\mathrm{un}}$, and let $\mathcal{P}_{\mathrm{un}}=\mathcal{P}_{\mathrm{un}, \mathcal{F}}$ be the corresponding parahoric group scheme for $G_{/ K_{\mathrm{un}}}$. There is a smooth $\mathscr{A}$-group scheme $\mathcal{P}$ whose generic fiber $\mathcal{P} / K$ may be identified with $G$ for which $\mathcal{P}_{\text {un }}=\mathcal{P} \otimes_{\mathscr{A}} \mathscr{A}_{\text {un }}$.

Proof. It follows from [BrTi 84, 4.6.30] that the action of $\Sigma$ on $K_{\text {un }}\left[G_{/ K_{\text {un }}}\right]=K[G] \otimes_{K} K_{\text {un }}$ leaves invariant the subalgebra $\mathscr{A}_{\text {un }}\left[\mathcal{P}_{\text {un }}\right.$ ], the coordinate algebra of $\mathcal{P}_{\text {un }}$. Thus [BrTi 84, 5.1.8] shows that $\mathcal{P}_{\text {un }}$ arises by base-change $\mathscr{A} \rightarrow \mathscr{A}$ un from a smooth $\mathscr{A}$-group scheme $\mathcal{P}$.

The parahoric group schemes associated with $G$ are precisely the $\mathscr{A}$-group schemes obtained via (6.5.1)] see [BrTi 84, §5.2]. 
Let $\mathcal{P}_{\text {un }}=\mathcal{P} \otimes_{\mathscr{A}} \mathscr{A}$ un be a parahoric group scheme corresponding to a $\Sigma$-invariant facet in $A_{\text {un. }}$. The special fiber $\mathcal{P}_{\text {un }} / k_{\text {alg }}$ of $\mathcal{P}_{\text {un }}$ is a linear algebraic group over $k_{\text {alg, }}$, and the special fiber $\mathcal{P}_{/ k}$ of $\mathcal{P}$ is a linear algebraic group over $k$. We now have:

(6.5.2). The special fibers of $\mathcal{P}_{\mathrm{un}}$ and $\mathcal{P}$ are related by $\mathcal{P}_{\mathrm{un} / k_{\mathrm{alg}}}=\left(\mathcal{P}_{/ k}\right) / k_{\mathrm{alg}}$.

6.6. Levi factors for the special fiber of $\mathcal{P}$. We prove in this section the Theorem stated in the introduction:

Proof of Theorem $A$. We first consider the case where $K=K_{\text {un }}$ and $G$ is split over $K$. Let $\mathcal{P}$ be a parahoric group scheme associated with $G$. Up to isomorphism of $\mathscr{A}$-group schemes, $\mathcal{P}$ arises via the construction in $\$ 6.2$

Since $k=k_{\mathrm{alg}}$, (6.3.2) shows that the special fiber $\mathcal{P}_{/ k}$ satisfies condition (L). Moreover, by (6.2.3) $\mathcal{P}_{/ k}$ satisfies the hypotheses of Theorem 5.5 It follows that $\mathcal{P}_{/ k}$ has a unique Levi factor containing a fixed maximal torus $T$. In particular, since $k=k_{\text {alg }}$ it follows that any two Levi factors of $\mathcal{P}_{/ k}$ are conjugate by an element of $\mathcal{P}_{/ k}(k)$.

Returning to the general case, the parahoric group scheme $\mathcal{P}$ associated with $G$ arises by étale descent from a parahoric $\mathscr{A}_{\text {un }}$-group scheme $\mathcal{P}_{\text {un }}$ associated with $G_{/ K_{\text {un }}}$ (6.5.1); thus $\mathcal{P}_{\text {un }}=\mathcal{P} \otimes_{\mathscr{A}} \mathscr{A}_{\text {un }}$ and $\mathcal{P}_{\text {un } / k_{\text {alg }}}=\mathcal{P}_{/ k} \otimes_{k} k_{\text {alg }}=\mathcal{P}_{/ k_{\text {alg }}}$.

Thus for any maximal $k$-torus $T$ of $\mathcal{P}_{/ k}$, the result above for $\mathcal{P}_{\text {un } / k_{\text {alg }}}$ together with (5.5.1) shows that $\mathcal{P}_{/ k}$ has a unique Levi factor (over $k$ ) containing $T$.

If $G$ is split over $K$, then $\mathcal{P}_{/ k}$ has a maximal torus which is $k$-split, and any Levi factor of $\mathcal{P} / k$ contains a maximal split torus. The existence assertions in parts (i) and (ii) of the Theorem now follow at once. A result of Borel-Tits shows that any two maximal split tori of $\mathcal{P} / k$ are conjugate by an element of $\mathcal{P}(k)$ [CGP 10, Theorem C.2.3] or [Sp 98, 15.2.6]; the conjugacy assertion in (i) now follows. The geometric conjugacy assertion in (ii) follows from the observation that all maximal tori of $\mathcal{P}_{/ k}$ are geometrically conjugate.

\section{EXAMPLES}

7.1. Cohomological construction of a linear algebraic group with no Levi decomposition. For a reductive group $G$, the non-vanishing of some $H^{2}(G,-)$ may be used to construct linear algebraic groups having no Levi factor. We give here an example.

Let $k$ be algebraically closed field of characteristic $p \geq 3$, and let $G=\mathrm{SL}_{3 / k}$. Write $T$ for the diagonal maximal torus in $G$, and $B$ for the Borel subgroup of lower triangular matrices.

Let $\alpha_{1}, \alpha_{2} \in X^{*}(T)$ be the simple roots of $G$ for the choice of $B$ (the weights of $T$ on $\operatorname{Lie}(B)$ are non-positive), and let $\omega_{1}, \omega_{2} \in X^{*}(T)$ be the fundamental dominant weights corresponding to the $\alpha_{i}$.

(7.1.1). If $\tau$ is a dominant weight for which $\left\langle\tau, \alpha_{1}^{\vee}+\alpha_{2}^{\vee}\right\rangle \leq p-2$, then $\operatorname{rad} V(\tau)=0$ so that $L(\tau)=V(\tau)=H^{0}(\tau)$.

Proof. The condition means that $\tau$ is in the closure of the lowest alcove for the dot-action of the affine Weyl group $W_{p}$ on $X^{*}(T)$; see [Ja 03, II.6.2(6)]. Since that alcove closure is a fundamental domain for the indicated action, one knows for any dominant weight $\gamma \leq \tau$ that $\gamma \notin W_{p} \bullet \tau$; thus by (2.4.1) and the linkage principle [Ja 03, II.6.17], $\operatorname{Hom}_{G}(\operatorname{rad} V(\tau), L(\gamma)) \simeq$ $\operatorname{Ext}_{G}^{1}(L(\tau), L(\gamma))=0$. Since $\tau$ is the highest weight of $V(\tau)$, it follows that $\operatorname{rad} V(\tau)=0$ as required.

According to [Ja 03, II.8.19], the G-module $V(\tau)$ has a filtration

$$
V(\tau)=V^{0} \supset V^{1} \supset V^{2} \supset \cdots
$$

for which $V^{1}=\operatorname{rad} V(\tau)$ and $J(\tau)=\sum_{i \geq 1} \mathrm{ch} V^{i}$ is given by the Jantzen sum formula [Ja 03, II.8.19]. The following result follows from (2.4.3) 
(7.1.2). Let $\tau$ be a dominant weight and suppose that $J(\tau)=\operatorname{ch} L$ for a simple G-module L. Then $\operatorname{rad} V(\tau) \simeq L$.

We are going to apply the Jantzen sum formula to achieve some cohomology computations. Let

$$
\lambda=p \omega_{1}, \quad \mu=(p-2) \omega_{1}+\omega_{2}, \quad \gamma=(p-3) \omega_{1} .
$$

(7.1.3). $\operatorname{rad} V(\mu)=L(\gamma)$, and $\operatorname{ch} L(\mu)=\chi(\mu)-\chi(\gamma)$.

Proof. Evaluating the Jantzen sum formula, we find that $J(\mu)=\chi\left(s_{\alpha_{1}+\alpha_{2}, p} \bullet \mu\right)=\chi(\gamma)$. Since $\left\langle\gamma, \alpha_{1}^{\vee}+\alpha_{2}^{\vee}\right\rangle=p-3$, (7.1.1) shows that $V(\gamma)=L(\gamma)$ so that $\operatorname{ch} L(\gamma)=\chi(\gamma)$. The assertion now follows from (7.1.2)

(7.1.4). $\operatorname{rad} V(\lambda)=L(\mu)$, and $\operatorname{ch} L(\lambda)=\chi(\lambda)-\chi(\mu)+\chi(\gamma)$.

Proof. Evaluating the Jantzen sum formula and using (7.1.3), we find that

$$
J(\mu)=\chi\left(s_{\alpha_{1}, p} \bullet \lambda\right)+\chi\left(s_{\alpha_{1}+\alpha_{2}, p} \bullet \lambda\right)=\chi(\mu)-\chi(\gamma)=\operatorname{ch} L(\mu),
$$

so the result follows from (7.1.2)

(7.1.5). $\operatorname{dim} \operatorname{Ext}_{G}^{2}(L(\lambda), L(\gamma))=1$.

Proof. Since $L(\gamma)=H^{0}(\gamma)$, it follows from (2.4.2) that $\operatorname{Ext}_{G}^{i}(V(\lambda), L(\gamma))=0$ for $i \geq 1$. Using (7.1.3) and (7.1.4) together with (2.4.1)] we find that $\operatorname{dim} \operatorname{Ext}_{G}^{1}(L(\mu), L(\gamma))=1$ and $\operatorname{Ext}_{G}^{1}(V(\lambda), L(\gamma))=0$. Now apply $\operatorname{Hom}_{G}(-, L(\gamma))$ to the short exact sequence $0 \rightarrow L(\mu) \rightarrow$ $V(\lambda) \rightarrow L(\lambda) \rightarrow 0$ to get an exact sequence

$$
0=\operatorname{Ext}_{G}^{1}(V(\lambda), L(\gamma)) \rightarrow \operatorname{Ext}_{G}^{1}\left(L(\mu), L(\gamma) \stackrel{\partial}{\rightarrow} \operatorname{Ext}_{G}^{2}\left(L(\lambda), L(\gamma) \rightarrow \operatorname{Ext}^{2}(V(\lambda), L(\gamma))=0 ;\right.\right.
$$

then $\partial$ is an isomorphism and the claim follows.

(7.1.6). There is a linear algebraic group $E$ with reductive quotient $\mathrm{SL}_{3}$, with abelian unipotent radical $W$ of dimension $\frac{3}{2}(p-1)(p-2)$, and with no Levi decomposition.

Proof. Write $W=L(\lambda)^{\vee} \otimes L(\gamma)$; using Steinberg's tensor product theorem [Ja 03, II.3.17], we observe that $W \simeq L\left(\omega_{2}\right)^{[1]} \otimes L\left((p-3) \omega_{1}\right)$; the Weyl degree formula together with[(7.1.1) now shows that $W$ has the indicated dimension. According to (7.1.5), we may choose a non-zero cohomology class

$$
[\alpha] \in H^{2}(G, W)=H^{2}\left(G, L(\lambda)^{\vee} \otimes L(\gamma)\right) \simeq \operatorname{Ext}_{G}^{2}(L(\lambda), L(\gamma))
$$

for $\alpha \in Z^{2}(G, W)$; the extension $E=E_{\alpha}$ of $G$ by $W$ then has the required properties.

7.2. Quasi-split unitary groups in even dimension. As in $₫$ let $\mathscr{A}$ be a Henselian DVR with fractions $K$ and residues $k$. We suppose in addition that the residue field $k$ is algebraically closed and has characteristic $p \neq 2$. In particular, any finite extension of $K$ is totally ramified over $K$.

We are going to give an example of a non-split reductive group $G$ over $K$ and a parahoric group scheme $\mathcal{P}$ over $\mathscr{A}$ associated with $G$ for which the special fiber $\mathcal{P} / k$ has a Levi decomposition, but for which there are Levi factors not conjugate by an element of $\mathcal{P}(k)$.

Let $L / K$ be a quadratic (hence Galois) extension. Denote the action of the non-trivial element $\tau \in \Gamma=\operatorname{Gal}(L / K)$ by $\left(x \mapsto x^{\tau}\right)$. Write $\mathscr{B}$ for the integral closure of $\mathscr{A}$.

(7.2.1). $\mathscr{B} \otimes \mathscr{A} k \simeq k[\varepsilon]$ where $k[\varepsilon]$ is the k-algebra of dual numbers with basis $1, \varepsilon$ for which $\varepsilon^{2}=0$. The action of $\tau$ on $\mathscr{B}$ induces the automorphism $\varepsilon \mapsto-\varepsilon$ on $k[\varepsilon]=\mathscr{B} \otimes_{\mathscr{A}} k$.

Proof. Indeed, $\mathscr{B}$ is generated as $\mathscr{A}$-algebra by a uniformizer which satisfies an Eisenstein equation of degree 2 over $\mathscr{A}$. 
Let $V$ be a $2 n$ dimensional $L$-vector space, and let $h: V \times V \rightarrow L$ be a non-degenerate skew-hermitian form on $V$. Let $G=\mathrm{SU}(V, h)$ be the corresponding special unitary group; thus $G$ is a $K$-form of $\mathrm{SL}_{2 n}$.

The fact that $G$ is quasisplit (6.4.1) means here that $h$ has maximal index; thus writing $I=\{ \pm 1, \pm 2, \ldots, \pm n\}$, we may find a basis $\left\{e_{i} \mid i \in I\right\}$ of $V$ such that

$$
h(v, w)=\sum_{i=1}^{n}\left(v_{i}\right)^{\tau} w_{-i}-\left(v_{-i}\right)^{\tau} w_{i}
$$

where we have written e.g. $v=\sum_{i \in I} v_{i} e_{i}$ for $v_{i} \in L$.

Consider the lattice $\mathscr{L}=\sum_{i \in I} \mathscr{B} e_{i} \subset V$ in $V$. Note that $h$ determines a skew hermitian form $\mathscr{L} \times \mathscr{L} \rightarrow \mathscr{B}$. Viewing $\mathscr{L}$ as an $\mathscr{A}$-lattice in the $K$-vector space $V=R_{L / K} V$, we may consider the schematic closure $\mathcal{P}$ [BrTi 84, I.2.6] of $G$ in the $\mathscr{A}$-group scheme GL(L) ; put another way, $\mathcal{P}$ is the $\mathscr{A}$-structure on $G$ defined by the $\mathscr{A}$-lattice $\mathscr{L}$.

(7.2.2). $\mathcal{P}$ is a parahoric group scheme for $G$.

Proof. Argue as in [Ti 77, §3.11], where instead the special unitary groups for odd dimensional $L$-vector spaces are treated in detail.

Write $M=\mathscr{L} \otimes_{\mathscr{A}} k$; using (7.2.1) we view $M$ as a free $k[\varepsilon]=\mathscr{B} \otimes_{\mathscr{A}} k$-module of rank $2 n$. Write $\beta$ for the form $\beta=h \otimes 1$; one sees that

$$
\beta: M \times M \rightarrow k[\varepsilon]
$$

is skew hermitian for the involution of $k[\varepsilon]$ defined by $\varepsilon \mapsto-\varepsilon$. Write $X \mapsto \sigma(X)$ for the involution (i.e. anti-automorphism of order 2) of $\operatorname{End}_{k[\varepsilon]}(M)$ determined by $\beta$; thus $\beta(X v, w)=$ $\beta(v, \sigma(X) w)$ for all $v, w \in M$.

The special fiber $\mathcal{P}_{/ k}$ identifies with the closed subgroup $H=S U\left(\operatorname{End}_{k[\varepsilon]}(M), \sigma\right)$ of the linear $k$-group $\operatorname{Aut}_{k[\varepsilon]}(M)=\operatorname{End}_{k[\varepsilon]}(M)^{\times}$defined by

$$
H(\Lambda)=\mathrm{SU}\left(\operatorname{End}_{k[\varepsilon]}(M), \sigma\right)(\Lambda)=\left\{x \in \operatorname{End}_{k[\varepsilon]}(M) \otimes_{k} \Lambda \mid \sigma(x) x=1, \operatorname{det}(x)=1\right\}
$$

for each commutative $k$-algebra $\Lambda$.

Since $\mathcal{P}$ is smooth over $\mathscr{A}$, the group scheme $H=\mathcal{P}_{/ k}$ is smooth over $k$ - i.e. $H$ is a linear algebraic group over $k$; of course, the smoothness of $H$ can be checked directly from its definition.

The bilinear form $\bar{\beta}$ induced on $\bar{M}=M / \varepsilon M$ from $\beta$ by reduction $\bmod \varepsilon$ is non-degenerate and symplectic. The involution $\bar{\sigma}$ induced on $\operatorname{End}_{k}(\bar{M})$ by $\sigma$ is evidently the adjoint involution of $\bar{\beta}$. Consider the natural map $\pi: \operatorname{Aut}_{k[\varepsilon]}(M) \rightarrow \mathrm{GL}(\bar{M})$ of linear algebraic groups over $k$.

(7.2.3). $\pi_{\mid H}$ defines a separable surjection $\pi_{\mid H}: H \rightarrow \operatorname{Sp}(\bar{M})=\operatorname{Sp}(\bar{M}, \bar{\beta}) \subset \operatorname{GL}(\bar{M})$ onto the symplectic group of $\bar{\beta}$.

Proof. It is clear that the image of $\pi$ lies in $\operatorname{Sp}(\bar{M})$. The Lie algebra of $H$ is

$$
\operatorname{Lie}(H)=\mathfrak{h}=\left\{X \in \operatorname{End}_{k[\varepsilon]}(M) \mid \operatorname{tr}(X)=0, \quad X+\sigma(X)=0\right\} .
$$

where $\operatorname{tr}(X) \in k[\varepsilon]$ is the trace. Given $\bar{Y} \in \mathfrak{s p}(\bar{M}, \bar{\beta})$, let $Y=i(\bar{Y}) \in \operatorname{End}_{k[\varepsilon]}(M)$ where we have written $i: \operatorname{End}_{k}(\bar{M}) \rightarrow \operatorname{End}_{k[\varepsilon]}(M)$ for the mapping determined by the $k$-linear splitting of the surjection $k[\varepsilon] \rightarrow k$. Then $Y \in \mathfrak{h}$ so that $d \pi_{\mid \mathfrak{h}}$ is surjective. Since $\operatorname{Sp}(\bar{M})$ is connected, $\pi_{\mid H}$ is indeed surjective.

Write

$$
\operatorname{Sym}\left(\operatorname{End}_{k}(\bar{M})\right)=\operatorname{Sym}\left(\operatorname{End}_{k}(\bar{M}), \bar{\sigma}\right)=\left\{X \in \operatorname{End}_{k}(\bar{M}) \mid X=\bar{\sigma}(X)\right\}
$$


for the space of symmetric endomorphisms of $\bar{M}$, and

$$
\operatorname{Sym}^{0}\left(\operatorname{End}_{k}(\bar{M})\right)=\left\{X \in \operatorname{Sym}\left(\operatorname{End}_{k}(\bar{M})\right) \mid \operatorname{tr}(X)=0\right\} .
$$

for those of trace 0. Conjugation makes $\operatorname{Sym}^{0}\left(\operatorname{End}_{k}(\bar{M})\right) \subset \operatorname{Sym}\left(\operatorname{End}_{k}(\bar{M})\right)$ into $\operatorname{Sp}(\bar{M})$ modules.

(7.2.4). (a) The kernel of $\pi_{\mid H}$ is the unipotent radical $R$ of $H$.

(b) There is an $\operatorname{Sp}(\bar{M})$-equivariant isomorphism $\operatorname{Sym}^{0}\left(\operatorname{End}_{k}(\bar{M})\right) \stackrel{\sim}{\rightarrow} R$; in particular, $R$ is a vector group and is linearizable for the action of $\operatorname{Sp}(\bar{M})$.

Proof. Let $R=\operatorname{ker} \pi_{\mid H}$. Then (7.2.3) shows that $R$ is smooth and that $H / R \simeq \operatorname{Sp}(\bar{M})$ is reductive, (a) will follow once we know that $R$ is connected and unipotent; thus it is enough to prove (b).

Since $R$ is smooth and $k$ is algebraically closed, it is enough to give an isomorphism on $k$-points. A $k$-point $y$ of $\operatorname{ker} \pi_{\mid H}$ has the form $y=1+\varepsilon X$ for $X \in \operatorname{End}_{k}(\bar{M})$. Then $y \in H(k)$ implies

$$
1=y \sigma(y)=(1+\varepsilon X) \sigma(1+\varepsilon X)=(1+\varepsilon X)(1-\varepsilon \bar{\sigma}(X))=1+\varepsilon(X-\bar{\sigma}(X)) .
$$

so that $X=\bar{\sigma}(X)$. Since $1=\operatorname{det}(y)=1+\varepsilon \operatorname{tr}(X)$ we deduce that $X \in \operatorname{Sym}^{0}\left(\operatorname{End}_{k}(\bar{M})\right)$. Thus $X \mapsto 1+\varepsilon X$ is the required isomorphism.

(7.2.5). Write $W=\operatorname{Sym}\left(\operatorname{End}_{k}(\bar{M})\right)$ and $W^{0}=\operatorname{Sym}^{0}\left(\operatorname{End}_{k}(\bar{M})\right)$. Then

(a) $H^{i}(\operatorname{Sp}(\bar{M}), W)=0$ for $i \geq 1$.

(b) $H^{i}\left(\operatorname{Sp}(\bar{M}), W^{0}\right)=0$ for $i \geq 2$.

(c) $H^{1}\left(\operatorname{Sp}(\bar{M}), W^{0}\right)= \begin{cases}0 & \text { if } n \not \equiv 0(\bmod p) \\ k & \text { if } n \equiv 0(\bmod p) \text {. }\end{cases}$

Proof. The $\operatorname{Sp}(\bar{M})$-module $W$ identifies with $\bigwedge^{2} \bar{M}$, which has a filtration by standard modules; cf. [Mc 98, Lemma 4.8.2] and recall the terminology from $\$ 2.4$. Thus (a) follows from (2.4.1)

Moreover, when $p$ does not divide $n$, the result just cited shows that $W$ is completely reducible, and that $W^{0}$ is a simple standard module (in fact, $W^{0}=V\left(\omega_{2}\right)=H^{0}\left(\omega_{2}\right)$ where $\omega_{2}$ is a certain fundamental dominant weight). Assertions (b) and (c) follow in this case from another application of (2.4.1).

Finally, suppose that $p$ divides $n$. Then the identity endomorphism of $\bar{M}$ is symmetric and has trace $2 n=0$ in $k$, so that $W^{0}$ has a trivial submodule. The result of loc. cit. shows that $W^{0}$ is a Weyl module (again, $W^{0}=V\left(\omega_{2}\right)$ ) which has composition length 2 , and there is a short exact sequence $0 \rightarrow W^{0} \rightarrow W \rightarrow k \rightarrow 0$ of $\operatorname{Sp}(\bar{M})$-modules. The resulting long exact sequence together with (a) now gives (b) and (c) in this case.

Proposition. (a) $H=\mathcal{P}_{/ k}$ has a Levi factor.

(b) If $n \not \equiv 0(\bmod p)$, and if $L, L^{\prime}$ are Levi factors of $H$, then $L=g L^{\prime} g^{-1}$ for some $g \in H(k)$.

(c) If $n \equiv 0(\bmod p)$, there are Levi factors $L, L^{\prime}$ of $H=\mathcal{P}_{/ k}$ which are not conjugate by an element of $H(k)$.

Proof. In view of (7.2.4) and (7.2.5), assertion (a) follows from Theorem 5.2 assertion (b) follows from Theorem [5.1, and assertion (c) follows from (4.5.2).

\section{REFERENCES}

[Bo 91] Armand Borel, Linear Algebraic Groups, 2nd ed., Grad. Texts in Math., vol. 126, Springer Verlag, 1991.

[BS68] A. Borel and T. A. Springer, Rationality properties of linear algebraic groups. II, Tôhoku Math. J. (2) 20 (1968), $443-497$.

[BoTi 65] Armand Borel and Jacques Tits, Groupes Réductifs, Publ. Math. IHES 27 (1965), 55-151. 
[Bou 02] Nicolas Bourbaki, Lie groups and Lie algebras. Chapters 4-6, Elements of Mathematics (Berlin), SpringerVerlag, Berlin, 2002. Translated from the 1968 French original by Andrew Pressley.

[Br 94] Kenneth S. Brown, Cohomology of groups, Graduate Texts in Mathematics, vol. 87, Springer-Verlag, New York, 1994. Corrected reprint of the 1982 original.

[BrTi 72] F. Bruhat and J. Tits, Groupes réductifs sur un corps local, Inst. Hautes Études Sci. Publ. Math. 41 (1972), 5-251 (French).

[BrTi 84] Groupes réductifs sur un corps local. II. Schémas en groupes. Existence d'une donnée radicielle valuée, Inst. Hautes Études Sci. Publ. Math. 60 (1984), 197-376 (French).

[CGP 10] Brian Conrad, Ofer Gabber, and Gopal Prasad, Pseudo-reductive groups, New Mathematical Monographs, vol. 17, Cambridge University Press, 2010.

[DeGa 70] Michel Demazure and Pierre Gabriel, Groupes algébriques. Tome I: Géométrie algébrique, généralités, groupes commutatifs, Masson \& Cie, Éditeur, Paris, 1970 (French).

[Hu 67] J. E. Humphreys, Existence of Levi factors in certain algebraic groups, Pacific J. Math. 23 (1967), 543-546.

[Jac 79] Nathan Jacobson, Lie algebras, Dover Publications Inc., New York, 1979. Republication of the 1962 original.

[Ja 03] Jens Carsten Jantzen, Representations of algebraic groups, 2nd ed., Mathematical Surveys and Monographs, vol. 107, American Mathematical Society, Providence, RI, 2003.

[Ja 97] Low-dimensional representations of reductive groups are semisimple, Algebraic groups and Lie groups, Austral. Math. Soc. Lect. Ser., vol. 9, Cambridge Univ. Press, Cambridge, 1997, pp. 255-266.

[Mc 03] George J. McNinch, Faithful representations of $\mathrm{SL}_{2}$ over truncated Witt vectors, J. Algebra 265 (2003), no. 2, 606-618.

[Mc 98] _ Dimensional criteria for semisimplicity of representations, Proc. London Math. Soc. (3) 76 (1998), no. 1, 95-149.

[Oe 84] Joseph Oesterlé, Nombres de Tamagawa et groupes unipotents en caractéristique p, Invent. Math. 78 (1984), no. 1, 13-88 (French).

[Pr 01] Gopal Prasad, Galois-fixed points in the Bruhat-Tits building of a reductive group, Bull. Soc. Math. France 129 (2001), no. 2, 169-174 (English, with English and French summaries).

[PR 84] Gopal Prasad and M. S. Raghunathan, Topological central extensions of semisimple groups over local fields, Ann. of Math. (2) 119 (1984), no. 1, 143-201.

[Ro 63] Maxwell Rosenlicht, Questions of rationality for solvable algebraic groups over nonperfect fields, Ann. Mat. Pura Appl. (4) 61 (1963), 97-120 (English, with Italian summary).

[Ro 77] G. Rousseau, Immeubles des groupes réductifs sur les corps locaux, Thèse, Université de Paris-Sud (1977).

[Se 79] Jean-Pierre Serre, Local fields, Graduate Texts in Mathematics, vol. 67, Springer-Verlag, New York, 1979. Translated from the French by Marvin Jay Greenberg.

[Ser97] G Galois cohomology, Springer-Verlag, Berlin, 1997. Translated from the French by Patrick Ion and revised by the author.MR 98g:12007

[Se 88] _ Algebraic groups and class fields, Graduate Texts in Mathematics, vol. 117, Springer-Verlag, New York, 1988. Translated from the French.

[Sp 98] Tonny A. Springer, Linear algebraic groups, 2nd ed., Progr. in Math., vol. 9, Birkhäuser, Boston, 1998.

[Ti 77] J. Tits, Reductive groups over local fields, Automorphic forms, representations and L-functions (Proc. Sympos. Pure Math., Oregon State Univ., Corvallis, Ore., 1977), Part 1, Proc. Sympos. Pure Math., XXXIII, Amer. Math. Soc., Providence, R.I., 1979, pp. 29-69.

[We 94] Charles A. Weibel, An introduction to homological algebra, Cambridge Studies in Advanced Mathematics, vol. 38, Cambridge University Press, Cambridge, 1994.

Department of Mathematics, Tufts University, 503 Boston Avenue, Medford, MA 02155, USA

E-mail address: george.mcninch@tufts.edu, mcninchg@member.ams.org 